\title{
Low-level blast exposure disrupts gliovascular and neurovascular connections and induces a chronic vascular pathology in rat brain
}

Miguel A. Gama Sosa ${ }^{1,2,3^{*}}$ D, Rita De Gasperi ${ }^{2,3,4}$, Georgina S. Perez Garcia ${ }^{3,4,5}$, Gissel M. Perez ${ }^{4}$, Courtney Searcy ${ }^{2,4}$, Danielle Vargas ${ }^{2,4}$, Alicia Spencer ${ }^{2,4}$, Pierce L. Janssen ${ }^{3,6}$, Anna E. Tschiffely ${ }^{7}$, Richard M. McCarron ${ }^{7,8}$, Benjamin Ache ${ }^{9}$, Rajaram Manoharan ${ }^{9}$, William G. Janssen ${ }^{3,6}$, Susan J. Tappan ${ }^{10}$, Russell W. Hanson ${ }^{11}$, Sam Gandy 2,3,4,5,12,13, Patrick R. Hof $3,6,12,14$, Stephen T. Ahlers ${ }^{7}$ and Gregory A. Elder $2,3,5,12,15$

\begin{abstract}
Much concern exists over the role of blast-induced traumatic brain injury (TBI) in the chronic cognitive and mental health problems that develop in veterans and active duty military personnel. The brain vasculature is particularly sensitive to blast injury. The aim of this study was to characterize the evolving molecular and histologic alterations in the neurovascular unit induced by three repetitive low-energy blast exposures $(3 \times 74.5 \mathrm{kPa})$ in a rat model mimicking human mild TBI or subclinical blast exposure. High-resolution two-dimensional differential gel electrophoresis (2DDIGE) and matrix-assisted laser desorption/ionization (MALDI) mass spectrometry of purified brain vascular fractions from blast-exposed animals 6 weeks post-exposure showed decreased levels of vascular-associated glial fibrillary acidic protein (GFAP) and several neuronal intermediate filament proteins (a-internexin and the low, middle, and high molecular weight neurofilament subunits). Loss of these proteins suggested that blast exposure disrupts gliovascular and neurovascular interactions. Electron microscopy confirmed blast-induced effects on perivascular astrocytes including swelling and degeneration of astrocytic endfeet in the brain cortical vasculature. Because the astrocyte is a major sensor of neuronal activity and regulator of cerebral blood flow, structural disruption of gliovascular integrity within the neurovascular unit should impair cerebral autoregulation. Disrupted neurovascular connections to pial and parenchymal blood vessels might also affect brain circulation. Blast exposures also induced structural and functional alterations in the arterial smooth muscle layer. Interestingly, by 8 months after blast exposure, GFAP and neuronal intermediate filament expression had recovered to control levels in isolated brain vascular fractions. However, despite this recovery, a widespread vascular pathology was still apparent at 10 months after blast exposure histologically and on micro-computed tomography scanning. Thus, low-level blast exposure disrupts gliovascular and neurovascular connections while inducing a chronic vascular pathology.
\end{abstract}

Keywords: Animal model, Blast, Brain, Chronic, Gliovascular, Neurovascular, Rat, Vascular pathology

\footnotetext{
*Correspondence: miguel.gama-sosa@mssm.edu

${ }^{1}$ General Medical Research Service, James J. Peters Department of Veterans

Affairs Medical Center, 130 West Kingsbridge Road, Bronx, NY 10468, USA

2Department of Psychiatry, Icahn School of Medicine at Mount Sinai, One

Gustave Levy Place, New York, NY 10029, USA

Full list of author information is available at the end of the article
}

(C) This is a U.S. Government work and not under copyright protection in the US; foreign copyright protection may apply. 2019 Open Access This article is distributed under the terms of the Creative Commons Attribution 4.0 International License (http:// creativecommons.org/licenses/by/4.0/), which permits unrestricted use, distribution, and reproduction in any medium, provided you give appropriate credit to the original author(s) and the source, provide a link to the Creative Commons license, and indicate if changes were made. The Creative Commons Public Domain Dedication waiver (http://creativecommons.org/ publicdomain/zero/1.0/) applies to the data made available in this article, unless otherwise stated. 


\section{Introduction}

Traumatic brain injury (TBI) has long been a major cause of combat-related disability [25]. Public awareness of TBI in the military has increased recently due to events in Iraq and Afghanistan where 10-20\% of veterans returning from these conflicts experienced a TBI [28]. While military related TBIs in Iraq and Afghanistan resulted from various mechanisms due to the wide spread use of improvised explosive devices, blast-related TBIs were most common [28]. In humans high-pressure blast waves can cause extensive multi-organ trauma including severe systemic vascular and CNS injury [78, 91]. However, in combat settings such as Iraq and Afghanistan, lower level exposures producing mTBIs have been much more common [28]. While many veterans who suffered blast-related TBIs improve others exhibit chronic postconcussive and mental health related symptoms that are largely refractory to therapy $[25,50]$. TBI, in particular repetitive mTBI, has also been associated with the later development of neurodegenerative diseases $[23,25,35]$. In addition there is increasing concern over the potential adverse consequences of subclinical blast exposures, which are common for many service members in non-combat settings [12].

How blast damages the nervous system is incompletely understood. The high metabolic demand of the brain requires a tight coordination between neuronal activity and blood flow [40]. Blast injury is known to affect the cerebral vasculature [27]. High level blast exposure induces prominent vasospasm in humans [5] and animals [8] along with reduced cerebral perfusion and altered contractile properties of large arteries $[9,73,86]$. As in humans, acute high-level blast exposure has a prominent hemorrhagic component which in animals includes venous hemorrhages [7, 27]. Blast-induced vasospasm has been suggested to in addition initiate a phenotypic switch in vascular smooth muscle cells that causes long term vascular remodeling $[4,39]$.

Many studies have described blast-related vascular pathology $[1,7,18,19,27,31,32,37,42,46-48,51-53,55,58$, $61,68,69,72,73,76,80,84,87]$. At the functional level, acute blast exposure has been associated with increased vascular permeability and blood-brain barrier (BBB) breakdown. Multiple studies have described increases in $\mathrm{BBB}$ permeability as judged by leakage of IgG, Evans Blue or sodium-fluorescein low-molecular-weight tracers $[1,36,45,49,52,54,56,57,60,61,71,74$, 81, 83, 85, 90, 92, 93]. Acutely, blast exposure in mice produces microlesions in the BBB that are associated with aberrant expression of phosphorylated tau protein $[42,60]$. Much evidence also supports a mechanism whereby a blast wave striking the body causes indirect central nervous system injury through what has been referred to as a thoracic effect [13, 21, 27, 80].
The pathophysiological basis of blast-related vascular pathology remains incompletely understood. Morphological and functional data indicate that both large and small brain vessels are affected [27]. However, little is known about the molecular changes associated with these abnormalities. We have been studying a rat model of blast overpressure injury that mimics a repetitive low-level blast exposure similar to that which would be encountered in a human mTBI or subclinical blast exposure [2]. Under the conditions of exposure in our model, at the histological level the cerebral vasculature appears selectively vulnerable [31]. Here we show that blast injury disrupts gliovascular and neurovascular connections and is associated with a chronic vascular pathology. Because neuronal and astrocytic mechanisms control cerebral blood flow, disruption of gliovascular and neurovascular interactions should affect cerebral autoregulation at multiple levels.

\section{Material and methods Animals}

Adult male Long Evans hooded rats (250-350 g, 10 weeks of age; Charles River Laboratories International, Wilmington, MA, USA) were used. All studies involving animals were reviewed and approved by the Institutional Animal Care and Use Committees of the Walter Reed Army Institute of Research (WRAIR)/Naval Medical Research Center and the James J. Peters VA Medical Center. Studies were conducted in compliance with the Public Health Service policy on the humane care and use of laboratory animals, the NIH Guide for the Care and Use of Laboratory Animals, and all applicable Federal regulations governing the protection of animals in research.

\section{Blast overpressure exposure}

Rats were exposed to overpressure injury using the WRAIR shock tube, which simulates the effects of air blast exposure under experimental conditions. The shock tube has a 12-in. circular diameter and is a 19.5 $\mathrm{ft}$. long steel tube divided into a $2.5 \mathrm{ft}$. compression chamber that is separated from a $17 \mathrm{ft}$. expansion chamber. The compression and expansion chambers are separated by polyethylene Mylar ${ }^{\mathrm{TM}}$ sheets ( $\mathrm{Du}$ Pont Co., Wilmington, DE, USA) that control the peak pressure generated. The peak pressure at the end of the expansion chamber was determined by piezoresistive gauges specifically designed for pressure-time (impulse) measurements (Model 102 M152, PCB, Piezotronics, Inc., Depew, NY, USA). This apparatus has been used in multiple prior studies to deliver blast overpressure injury to rats $[2,15-17,22,26,31-33,38,64-66]$.

Individual rats were anesthetized using an isoflurane gas anesthesia system consisting of a vaporizer, gas lines and valves and an activated charcoal scavenging system 
adapted for use with rodents. Rats were placed into a polycarbonate induction chamber, which was closed and immediately flushed with 5\% isoflurane in air mixture for $2 \mathrm{~min}$. Rats were placed into a cone shaped plastic restraint device and then placed in the shock tube. Movement was further restricted during the blast exposure using $1.5 \mathrm{~cm}$ diameter flattened rubber tourniquet tubing. Three tourniquets were spaced evenly to secure the head region, the upper torso and lower torso while the animal was in the plastic restraint cone. The end of each tubing was threaded through a toggle and run outside of the exposure cage where it was tied to firmly affix the animal and prevent movement during the blast overpressure exposure without restricting breathing. Rats were randomly assigned to sham or blast conditions and were placed in the shock tube lying prone with the plane representing a line from the tail to the nose of the body in line with the longitudinal axis of the shock tube with the head placed more upstream. Further details of the physical characteristics of the blast wave are described in Ahlers et al. [2]. Blast exposed animals received $74.5 \mathrm{kPa}$ (equivalent to $10.8 \mathrm{psi}$, duration $4.8 \mathrm{~ms}$, impulse $175.8 \mathrm{kPa}$ *ms) exposures administered one exposure per day for three consecutive days. Sham exposed animals were treated identically including receiving anesthesia and being placed in the blast tube but did not receive a blast exposure. Within 10 days after the last blast or sham exposure animals were transported in a climate controlled van from the WRAIR to the James J. Peters VA Medical Center (Bronx, NY, USA). Animals left in the morning from the WRAIR and arrived in the afternoon of the same day at the James J. Peters VA Medical Center, where all other procedures were performed.

\section{Brain vascular isolation}

Rats were euthanized by $\mathrm{CO}_{2}$ inhalation, and the brain immediately dissected. Whole brains were cleaned of meninges and homogenized in cold 18\% dextran in phosphate-buffered saline (PBS, $10 \mathrm{ml} / \mathrm{g}$ of tissue) using a Potter-Elvejehm homogenizer with a loose-fit Teflon pestle (6-8 strokes at low speed, setting 2 of a Wheaton overhead stirrer, Millville, NJ, USA). Homogenization resulted in a thick homogenate of low density that was overlayed over an equal volume of Ficoll-Paque PLUS ${ }^{\text {ma }}$ (GE Healthcare Life Sciences, Marlborough, MA, USA) to form a single-step discontinuous gradient. Centrifugation was performed for $30 \mathrm{~min}$ at $1500 \times \mathrm{g}$ and $4{ }^{\circ} \mathrm{C}$. Pellets were then resuspended, washed twice with PBS and stored at $80^{\circ} \mathrm{C}$ for biochemical analyses or fixed in $4 \%$ paraformaldehyde (PFA) in PBS and kept at $4{ }^{\circ} \mathrm{C}$ until use.

\section{High-resolution two-dimensional differential gel electrophoresis}

Purified vascular preparations from brains of control and blast-exposed rats (two samples/group) isolated from control and blast exposed brains 6 weeks after exposure were compared by two-dimensional differential gel electrophoresis (2D-DIGE). 2D-DIGE and subsequent identification of candidate proteins by matrix-assisted laser desorption/ionization (MALDI) mass spectrometry (MS) were performed by Applied Biomics, Inc., (Hayward, CA, USA). Samples were extracted with 2D lysis buffer (30 mM Tris $\mathrm{HCl}, \mathrm{pH} 8.8,7$ $\mathrm{M}$ urea, $2 \mathrm{M}$ thiourea, $4 \%$ CHAPS,). Control and blast samples were labeled with $\mathrm{Cy} 2$ and $\mathrm{Cy} 5$ dyes, respectively, and mixed with $2 \mathrm{X} 2 \mathrm{D}$ sample buffer $(8 \mathrm{M}$ urea, $4 \%$ CHAPS, $20 \mathrm{mg} / \mathrm{ml} \mathrm{DTT,} 2 \%$ pharmalytes and trace bromophenol blue). DeStreak solution and rehydration buffer (7 M urea, $2 \mathrm{M}$ thiourea, $4 \%$ CHAPS, $20 \mathrm{mg} / \mathrm{ml}$ DTT, $1 \%$ pharmalytes and $0.01 \%$ bromophenol blue) were added. Proteins were separated by isoelectric focusing (IEF) (3-10) followed by sodium dodecyl sulfate (SDS)-polyacrylamide gel electrophoresis (PAGE). Gels were imaged with a Typhoon Trio Imager (GE Healthcare Life Sciences) and analyzed with the Image QuantTL software (GE Healthcare Life Sciences). Gel and cross-gel analyses were performed using DeCyder software (GE Healthcare Life Sciences) to calculate the fold-change in protein expression levels between control and blast samples.

\section{Protein identification by MS}

Candidates for protein identification were selected based on fold-change comparisons between control and blast samples and the calculated $p$-value. Spots of interest were selected using the Ettan Spot Picker (GE Healthcare) and in-gel digested with Trypsin Gold (Promega, Madison, WI, USA). The tryptic peptides were desalted with ZipTip C18 (Millipore, Billerica, MA, USA) and eluted with $5 \mathrm{mg} / \mathrm{ml} \alpha$-cyano-4-hydroxycinnamic acid in $25 \mathrm{mM}$ ammonium bicarbonate, $50 \%$ acetonitrile and $0.1 \%$ trifluoroacetic acid and spotted onto an AB SCIEX MALDI plate (AB Sciex, Framingham, MA, USA). MALDI-TOF MS and TOF/TOF (tandem MS/MS) were performed using a 5800 mass spectrometer (AB Sciex, Concord, Ontario, Canada). The peptide mass and associated fragmentation data were determined using the GPS Explorer Workstation equipped with a MASCOT search engine (Matrix Science, Boston, MA, USA). Searches were performed without constraining protein molecular weight or isoelectric point, with variable methionine oxidation and cysteine carbamidomethylation, and with one missed cleavage permitted. Candidates with an ion confidence interval $(\mathrm{CI} \%)$ or protein score $\mathrm{CI} \%>95$ were considered significant.

\section{Western blot analysis}

Vascular fractions were lysed in $10 \mathrm{mM} \mathrm{NaPO}$, $\mathrm{pH} 7.4$, $150 \mathrm{mM} \mathrm{NaCl}, 2 \mathrm{mM}$ EDTA, $1 \%$ Triton X-100, 0.5\% 
sodium deoxycholate, and 1\% SDS supplemented with protease and phosphatase inhibitor cocktails 2 and 3 (Sigma-Aldrich, St Louis, MO, USA). The lysates were centrifuged at $15000 \times \mathrm{g}$ for $15 \mathrm{~min}$, and the protein concentration in the supernatants was determined with the BCA reagent (ThermoFisher, Waltham MA, USA) according to the manufacturer's protocol. Proteins $(10-20 \mu \mathrm{g})$ were separated by SDS-PAGE, and the gels blotted onto polyvinylidene difluoride (PVDF) membranes. The membranes were blocked in a solution containing $50 \mathrm{mM}$ Tris- $\mathrm{HCl}, \mathrm{pH}$ 7.6, $0.15 \mathrm{M} \mathrm{NaCl}$ (TBS) and $0.5 \%$ non-fat dry milk and incubated overnight at $4{ }^{\circ} \mathrm{C}$ with primary antibody diluted in blocking solution. Membranes were incubated with the appropriate horseradish peroxidase-conjugated secondary antibody (1:5000-1:10,000, GE Healthcare Life Sciences) in blocking solution, and the bands visualized with the ECL Prime Western Blot detection reagent (GE Healthcare Life Sciences). The blots were imaged with the Amersham Imager 600 (GE Healthcare Life Sciences), and bands quantitated with Image QuantTL software (GE Healthcare Life Sciences). For analysis of GFAP expression, brain subregions were dissected from control and blast-exposed animals. Tissue lysates were analyzed as above except that $50 \mu \mathrm{g}$ of protein were loaded onto the gel.

The following primary antibodies and dilutions were used: rabbit polyclonal anti-glial fibrillary acidic protein (anti-GFAP, 1:2000, G9269), rabbit polyclonal anti-neurofilament heavy polypeptide (anti-NFH, 1:2000, N4142) and mouse monoclonal anti- $\alpha$-SMA $(1: 2000, \alpha$-smooth muscle actin clone 1A4, A25470; all from Sigma-Aldrich), rabbit polyclonal anti- $\alpha$-internexin (anti- $\alpha$-INX, 1:1000, GTX130053) and rabbit polyclonal anti-neurofilament medium polypeptide (anti-NFM, 1:1500, GTX133215; both from GeneTex, Irvine, CA, USA), rabbit polyclonal anti-Ephrin type $B$ receptor (EPHB4, 1:1000, 20,883-1-AP; Proteintech, Rosemont, IL, USA). A mouse monoclonal antibody against GAPDH (GeneTex, 1:4000, GTX627408) was used to assess GAPDH expression as a loading control.

\section{General histology and immunohistochemical analysis}

To prepare brain tissue for immunohistochemical evaluation, rats were anesthetized with $150 \mathrm{mg} / \mathrm{kg}$ ketamine and $30 \mathrm{mg} / \mathrm{kg}$ xylazine and perfused with cold $4 \%$ PFA in PBS. Brains were dissected, post-fixed for $48 \mathrm{~h}$ in $4 \%$ PFA and stored in sterile PBS at $4{ }^{\circ} \mathrm{C}$. Coronal sections were prepared at $50 \mu \mathrm{m}$ thickness with a VT1000S Vibratome (Leica Biosystems, Buffalo Grove, IL, USA). General brain histology was analyzed in hematoxylin/ eosin (H\&E)-stained sections.

For immunohistochemical analysis, sections were blocked with $10 \%$ normal goat serum in $50 \mathrm{mM}$ Tris $\mathrm{HCl}, \mathrm{pH} 7.6,0.15 \mathrm{M} \mathrm{NaCl}, 0.3 \%$ Triton-X-100 and incubated overnight with the primary antibodies diluted in blocking solution at room temperature. After washing with PBS (6 times for $10 \mathrm{~min}$ each), sections were incubated with the appropriate Alexa (488 and 568)-conjugated secondary antibodies (1:300, ThermoFisher) in blocking solution for $2 \mathrm{~h}$. After washing with PBS (6 times for $10 \mathrm{~min}$ each), the sections were mounted with Fluorogel mounting medium (Electron Microscopy Sciences, Hatfield, PA, USA). To visualize nuclei, sections were incubated in PBS containing $0.1 \mu \mathrm{g} / \mathrm{ml}$ DAPI (4',6-diamidino-2'-phenylindole dihydrochloride) in the next to last PBS wash. The primary antibodies were a rat monoclonal anti-GFAP (1:500; gift of Virginia Lee, University of Pennsylvania, Philadelphia PA, USA), rabbit anti-GFAP (1:500, G9269), rabbit anti-NFH(1:500, N4142), rabbit anti-laminin (1:300, L9393), mouse anti- $\alpha$-smooth muscle actin ( $\alpha$-SMA, 1:300, A2547) (all from Sigma-Aldrich), and mouse anti-phosphorylated NFH, (SMI31, 1:300, SMI 31P, BioLegend, San Diego, CA, USA). Primary negative controls consisted of omission of the primary antibodies. Images were collected with a Zeiss 700 confocal microscope (Jena, Germany) and processed with Adobe Photoshop CC (Adobe Systems, San Jose, CA, USA). Staining of the vasculature was performed with anti-collagen type IV antibodies (rabbit anti-rat collagen type IV, 1:300, ab6586, Abcam, Cambridge, MA, USA) as described elsewhere $[30,34]$. Briefly, sections were incubated with $1 \mathrm{mg} / \mathrm{ml}$ pepsin (Agilent Technologies, Santa Clara, CA, USA) in $3 \%$ acetic acid for $50 \mathrm{~min}$ at $37^{\circ} \mathrm{C}$, washed with PBS, blocked and stained with antibodies against collagen IV and GFAP in combination with the respective Alexa-conjugated secondary antibodies as described above. For immunostaining of purified brain vascular preparations, freshly resuspended vascular fractions were fixed with $4 \%$ PFA in PBS overnight, rinsed with PBS, blocked as above and incubated overnight with biotin-labeled Griffonia simplicifolia isolectin B4 $(0.3 \mu \mathrm{g} / \mathrm{ml}$, L3759, Sigma-Aldrich). After washing with PBS, Alexa-568-conjugated streptavidin (1:300, Thermo Fisher) was added and incubated for 2 $\mathrm{h}$ at room temperature. After washing with PBS, vessels were transferred onto a slide, mounted with FluoroGel mounting medium and imaged with a Zeiss 700 confocal microscope.

\section{Electron microscopy}

Electron microscopy (EM) was performed using protocols optimized to study the ultrastructure of the vasculature as previously described [14, 41, 44, 88]. Anesthetized rats were perfused as described above with cold $2 \%$ PFA containing $2 \%$ glutaraldehyde in $0.1 \mathrm{M}$ sodium phosphate buffer, $\mathrm{pH}$ 7.0. Tissue was removed and post-fixed in the same fixative overnight. Fixed brains were placed on a rat brain slicer matrix, and coronal slices containing the frontal cortex were excised and processed. Slices were 
cryoprotected by immersion in $4 \% \mathrm{D}$-glucose, followed by increasing concentrations of glycerol (from 10 to $30 \%$ in phosphate buffer; $v / v$ ) and plunged rapidly into liquid propane cooled with liquid nitrogen $\left(-190^{\circ} \mathrm{C}\right)$ in a Universal Cryofixation System KF80 (Reichert-Jung, Vienna, Austria). The samples were immersed in $0.5 \%$ uranyl acetate in anhydrous methanol $\left(-90^{\circ} \mathrm{C}, 24 \mathrm{~h}\right)$ in a cryosubstitution AFS unit (Leica, Vienna, Austria). The temperature was raised from $-90^{\circ} \mathrm{C}$ to $-45^{\circ} \mathrm{C}$ in steps of $4{ }^{\circ} \mathrm{C} / \mathrm{h}$. After washing with anhydrous methanol, the samples were infiltrated with Lowicryl HM20 resin (Electron Microscopy Sciences, Fort Washington, PA, USA) at $-45^{\circ} \mathrm{C}$. Polymerization with ultraviolet light $(360 \mathrm{~nm})$ was performed for $48 \mathrm{~h}$ at $-45^{\circ} \mathrm{C}$ and then for $24 \mathrm{~h}$ at $0{ }^{\circ} \mathrm{C}$. Ultrathin sections $(70 \mathrm{~nm})$ were cut with a diamond knife on a Reichert-Jung ultramicrotome and mounted on nickel grids using a Coat-Quick adhesive pen (Electron Microscopy Sciences). Sections were imaged on a Hitachi 7700 electron microscope (Tokyo, Japan) and photographed with an Advantage CCD camera (Advanced Microscopy Techniques, Danvers, MA, USA). Image brightness and contrast were adjusted using the Adobe Photoshop CC software. Images were analyzed by an investigator blinded to the condition of the animal.

\section{High-resolution micro-computed tomography (micro-CT) scanning}

Anesthetized rats were transcardially perfused with $250 \mathrm{ml}$ of a $30 \%$ solution of the Brite Vu contrast agent (Scarlet Imaging, Murray UT, USA) maintained at $65-70{ }^{\circ} \mathrm{C}$. The perfused animals were chilled by immersion in an ice-water bath for $60 \mathrm{~min}$ to gel the intravascular contrast agent. Brains were then dissected and post-fixed overnight in $4 \%$ PFA in PBS. Brains were scanned at a $7.5-\mu \mathrm{m}$ voxel size using $50 \mathrm{kVp}$ and $200 \mu \mathrm{A} \mathrm{X}$-ray settings with exposure time of $810 \mathrm{~ms}$ per frame, with 3 frames averaged at each projection angle with a Bruker SkyScan 1272 micro-CT (Microphotonics, Allentown, PA, USA). 3D reconstruction was performed with Bruker's CTVox 3D visualization software.

\section{Quantification of the cerebral vasculature}

Morphological profiling of the cerebral vasculature was performed with the Vesselucida 360 software (v2018.1.1, MBF Bioscience, Williston, VT, USA) using data obtained from the micro-CT scans and automatically reconstructing the respective $3 \mathrm{D}$ vascular networks. Automatic reconstruction of the vasculature was performed using identical settings for all animals using the voxel scooping algorithm with the following settings: trace and seed sensitivity set to 80 , medium seed density with refine filter set to 2 , maximum gap tolerance. No manual editing was performed. The vascular parameters determined were total length, total surface area and total volume. Analysis was conducted by an investigator blinded to the condition of the animal.

\section{Statistical analyses}

Statistical differences were evaluated with unpaired $t$-tests using Prism 7.0 software (GraphPad, La Jolla, CA). Statistical significance was set at an $\alpha$ level of 0.05 .

\section{Results}

A rat model of low-level blast injury

We have been studying an animal model developed to mimic a level of blast exposure that would be associated with human mild TBI or a subclinical exposure. Initial studies using this system established that exposures up to $74.5 \mathrm{kPa}$ (equivalent to $10.8 \mathrm{psi}$ ), caused no post-exposure apnea or mortality [2]. While representing a level of blast that is transmitted to the brain $[2,15]$, these exposures produced only mild transient behavioral disturbances and no obvious general brain histopathology. Histological examination of the lung showed no hemorrhages nor other pathology [2]. By contrast higher exposures (17.4 psi) led to frank subdural and intraparenchymal hemorrhages and visibly evident histopathology in brain along with pulmonary hemorrhages [2].

Because multiple blast exposures have been common among veterans returning from Iraq and Afghanistan [28], for most studies we used a design in which rats received three $74.5-\mathrm{kPa}$ exposures delivered one exposure per day on 3 consecutive days. In multiple studies using this design we confirmed the lack of neuronal pathology at the light and EM levels as well as the absence of any reactive astrocytosis or general inflammatory reaction $[2,22,26,31-33]$ despite the fact that these animals develop chronic PTSD-related behavioral traits [26, 64-67]. Thus based on our experience presented in a number of published studies we believe that these blast pressures mimic a low-level blast exposure equivalent to a human mTBI or subclinical blast exposure. Examples of H\&E stained sections from rats studied 6 weeks after blast exposure are shown in Fig. 1.

\section{Reduced GFAP expression and fewer astroglial attachments in isolated vascular fractions from blast- exposed rats at 6 weeks after blast exposure}

Despite the generally benign appearance of the brain seen at the level of standard histopathology, EM analyses revealed that the brain vasculature in this model is particularly sensitive to blast injury [31]. To explore molecular changes in the vasculature of blast-exposed rats, we devised a method to isolate enriched vascular fractions from rat brain. Examples of isolated brain vascular fractions from non-blast exposed adult Long-Evans rats are shown in Fig. 2. Microscopically enriched 

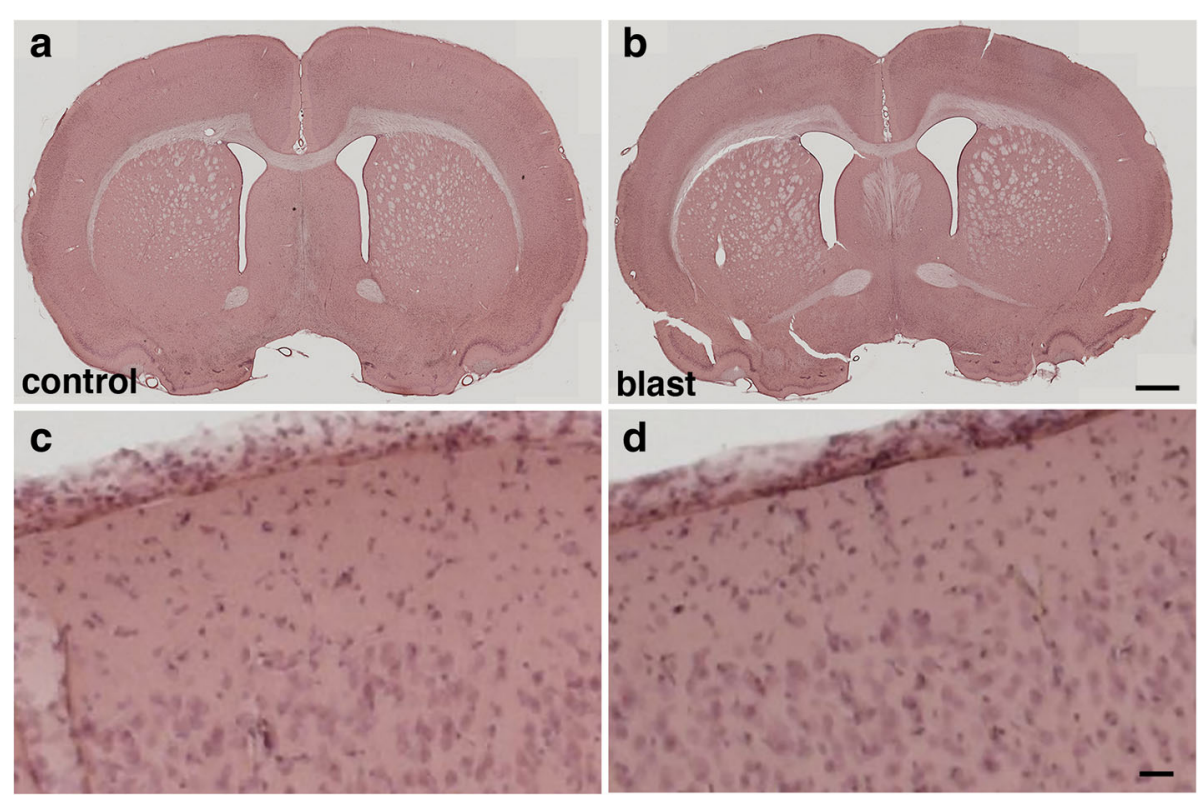

Fig. 1 Lack of overt histopathology in the brain at 6 weeks after the last blast exposure. a-d Sections from control (a, c) and blast-exposed (b, d) animals were stained with hematoxylin and eosin. Rats were euthanized 6 weeks after blast exposure. Higher power images of the frontal motor cortex are shown panels (c) and (d). Scale bars, $500 \mu \mathrm{m}(\mathbf{a}, \mathbf{b}) ; 40 \mu \mathrm{m}(\mathbf{c}, \mathbf{d})$
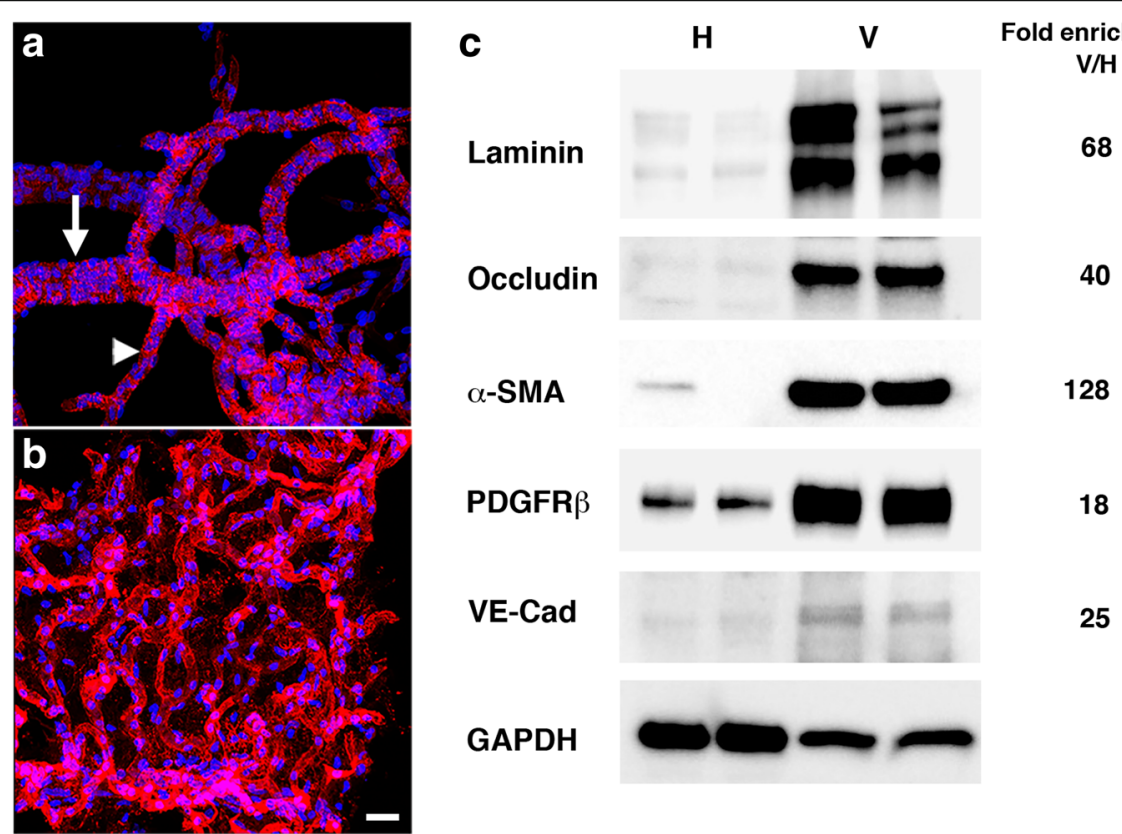

Fig. 2 Immunohistochemical and biochemical characterization of purified vascular fractions from rat brain. $\mathbf{a}-\mathbf{b}$ Isolated brain vascular fractions from a 3 month-old non-blast exposed rat is shown stained with Griffonia simplicifolia isolectin B4 (red). Nuclei were counterstained with DAPI (blue). a Muscular artery (indicated by an arrow) gives rise to a medium-sized vessel (indicated by an arrowhead). b Medium-and small-sized vessels and microvessels. Scale bar, $50 \mu \mathrm{m}$. c Immunoblots of total brain homogenates and vascular-enriched preparations are shown blotted with antibodies against endothelial (laminin, occludin, VE-cadherin), vascular smooth muscle (a-SMA), and pericyte (PDGFRß) markers. All lanes were loaded with $10 \mu \mathrm{g}$ of protein. Fold-enrichments in the vascular preparations vs. the whole brain homogenates are indicated on the right. Note enrichment of all the markers tested in the brain vascular fractions 
preparations contained a mixture of variously sized vessels linked in networks (vascular trees) that included arteries, arterioles, veins, venules and capillaries (Fig. 2a-b). Western blotting with antibodies against the vascular extracellular matrix protein laminin showed an average 68-fold enrichment (in two independent preparations) relative to the initial total brain homogenates (Fig. 2c). A similar enrichment of other vascular related components was demonstrated, including endothelial (occludin, 40-fold; VE-cadherin, 25-fold), pericyte (platelet-derived growth factor receptor $\beta$ [PDGFR $\beta$ ], 18-fold), and vascular smooth muscle ( $\alpha$-smooth muscle actin [ $\alpha$-SMA], 128-fold) markers (Fig. 2c).

Proteomic studies were conducted using purified brain vascular fractions isolated from control and blast-exposed rat brains at 6 weeks after the last of three consecutive daily blast exposures that were delivered when the animals were 10 weeks old. To determine the relative composition of the vascular fractions isolated from control and blast-exposed brains, we analyzed them by Western blotting with antibodies against $\alpha$-SMA (a preferential marker of arterial smooth muscle) and EPHB4 (a marker of venous endothelial cells) [24]. No differences were noted between vascular preparations from blast-exposed and control rats (Fig. 3) suggesting that the relative composition of arterial and venous elements isolated is not affected by blast exposure.

Protein profiles from two blast-exposed and two control animals were compared by 2D-DIGE (Fig. 4a). Using a cut-off level of 1.5 , a total of 77 proteins showed altered expression in vascular preparations from the blast-exposed compared with controls. Based on fold-change between blast-exposed and control as well as calculated $p$-values, 18 proteins were selected for identification by MS. This analysis identified 13 distinct proteins (Fig. 4b).

Surprisingly, the protein showing the most altered expression was GFAP, which was decreased in blast-exposed animals. To confirm the proteomic data, levels of GFAP in isolated vessels from control and blast-exposed animals were determined by Western blotting. As shown in Fig. 5a, Western blotting confirmed that GFAP expression was decreased (about 3-fold) in the isolated brain vascular fractions from blast-exposed rats.

The presence of GFAP in isolated vascular fractions suggested that astroglial endfeet were remaining attached to vessels, with the reduction of GFAP in blast-exposed samples reflecting loss of these attachments. To determine whether astroglial attachments could be visualized in isolated vascular fractions, we performed GFAP immunostaining (Fig. 5b-c). In controls (Fig. 5b), GFAP staining occurred in patches, consistent with perivascular attachment of astroglial endfeet. Others have also observed astrocytic endfeet attached to purified mouse vasculature [10, 20, 77]. In blast-exposed rats (Fig. 5c), perivascular GFAP staining also occurred in patches but was noticeably reduced compared to controls, consistent with astroglial endfeet being lost. To determine whether changes in GFAP levels could be detected in brain tissue we performed Western blotting on selected subregions (anterior cortex, posterior cortex, and hippocampus) harvested 6 weeks after blast exposure. As shown in Fig. 6, GFAP was decreased in the left anterior cortex as well as the left and right posterior cortex and the right hippocampus (about 1.7 fold).

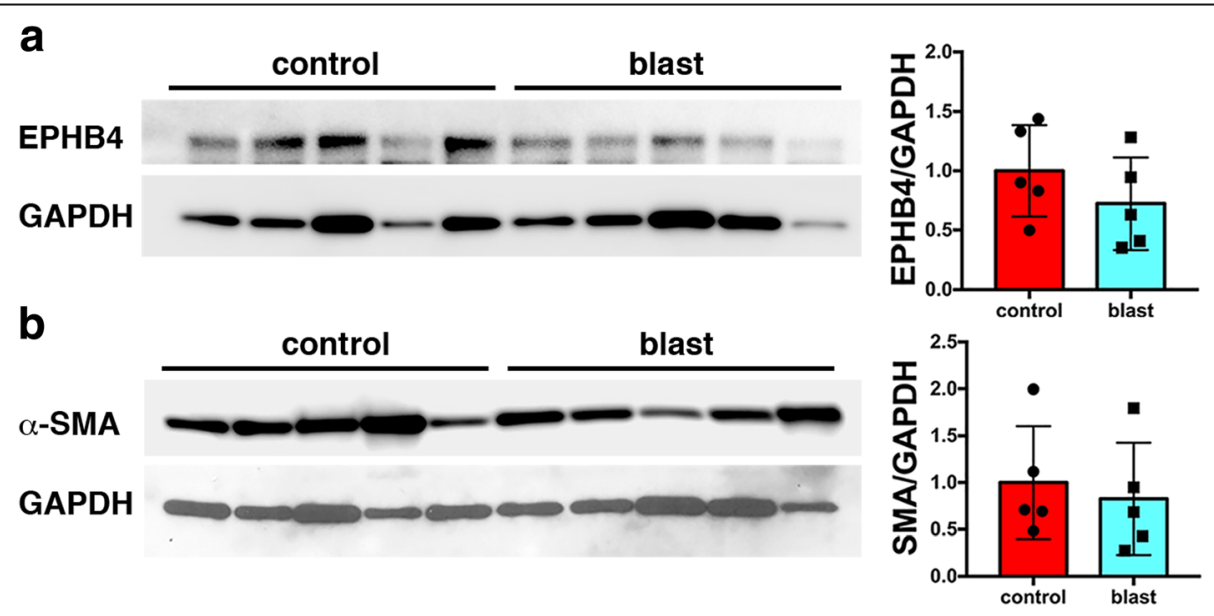

Fig. 3 Comparison of expression of venous and arterial markers in vessels isolated from control and blast-exposed rat brains 6 weeks after the final blast exposure. $\mathbf{a}-\mathbf{b}$ Western blotting showing expression of EPHB4 (a) and a-SMA (b) in isolated vascular fractions from five control and five blast-exposed animals. GAPDH was used as a loading control. All lanes were loaded with $10 \mu \mathrm{g}$ of protein and contain protein from separate animals. Quantitation of the blots is shown in the right panels with expression normalized to GAPDH. There were no statistically significant differences (unpaired t-tests, $n=5 /$ group) 


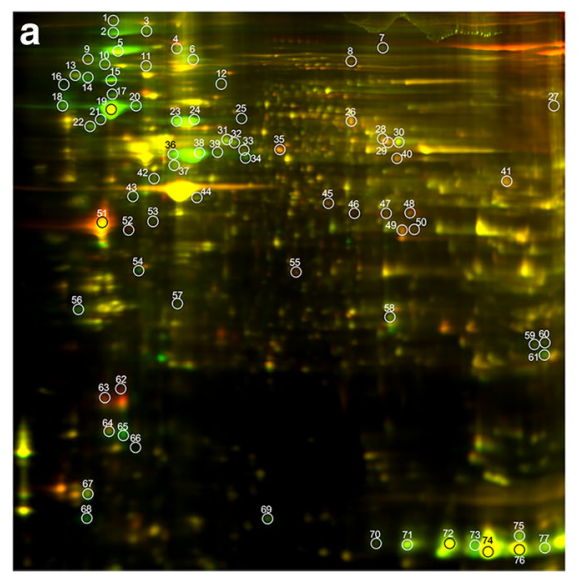

\begin{tabular}{ccc} 
b & & \multicolumn{2}{c}{$\begin{array}{c}\text { EXPRESSION RATIO } \\
\text { (blast/control) } \\
\text { p-value }\end{array}$} & $\begin{array}{c}\text { Averag } \\
\text { Ratio }\end{array}$ \\
& & -4.8 \\
2 & 0.008 & -4.8 \\
5 & 0.003 & -5.4 \\
10 & 0.006 & -2.1 \\
15 & 0.001 & -4.9 \\
19 & 0.000 & -6.0 \\
23 & 0.000 & -3.4 \\
24 & 0.001 & -3.9 \\
25 & 0.008 & -3.3 \\
31 & 0.010 & -2.2 \\
36 & 0.002 & -6.3 \\
43 & 0.004 & -2.9 \\
45 & 0.023 & 1.6 \\
46 & 0.032 & 2.5 \\
47 & 0.009 & 1.7 \\
58 & 0.014 & -1.6 \\
62 & 0.088 & 2.8 \\
67 & 0.016 & -1.5 \\
73 & 0.020 & -3.2
\end{tabular}

PROTEIN IDENTIFIED
ofilament heavy polypeptide,Nfh
ofilament medium polypeptide,Nfm
plasmin, Grp94
ofilament medium polypeptide,Nfm
ofilament light polypeptide, NfI
ternexin, Ina
ternexin, Ina
ofilament medium polypeptide,Nfm
fibrillary acidic protein, Gfap
fibrillary acidic protein, Gfap
CoA thioesterase, Acot7
hol dehydrogenase (NADP+), Akr1a1
e phosphate isomerase, TPI
related protein, Rap-2b
sin light polypeptide 6, Myl6
oglobin subunit beta, Hbb1

Fig. 4 Expression profile of Cy-dye labeled proteins in vascular fractions prepared from brains of blast-exposed and control animals 6 weeks after blast exposure. a Representative 2D-DIGE image showing overlay of proteins from isolated vascular fractions of control (Cy-3 labeled, green spots) and blastexposed (Cy-5 labeled, red spots) animals. White circles indicate differentially expressed proteins identified by image analysis with the DeCyder software. Each gel was loaded with $10 \mu \mathrm{g}$ of protein. b Table showing the 18 spots identified by MS which represent a total of 13 distinct proteins. ID numbers correspond to the spot number in the gel image in panel (a). Average fold changes and $p$-values in blast relative to control are indicated

Reduced neuronal intermediate filament protein levels in vascular preparations from blast-exposed rats at 6 weeks following blast exposure

Somewhat surprisingly, besides GFAP, 4 of the first 9 proteins identified by MS were intermediate filament proteins (IFs) (Fig. 4b), but rather than being glial or endothelial in origin they were neuronal-associated IFs $(\alpha-I N X$ and the light, medium and heavy polypeptide neurofilaments). All were decreased in blast-exposed vessels based on 2D-DIGE. Figure 7a shows Western

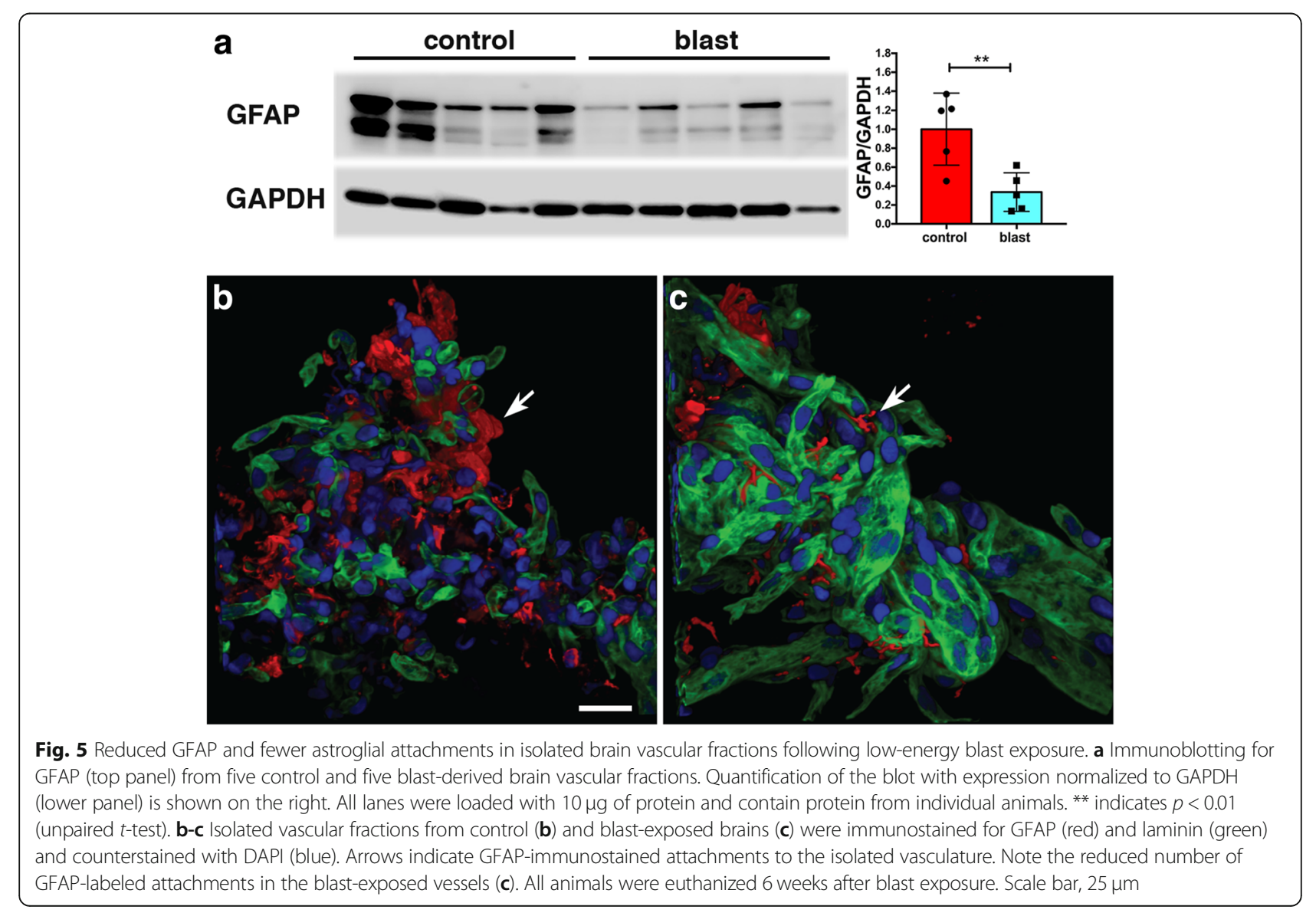




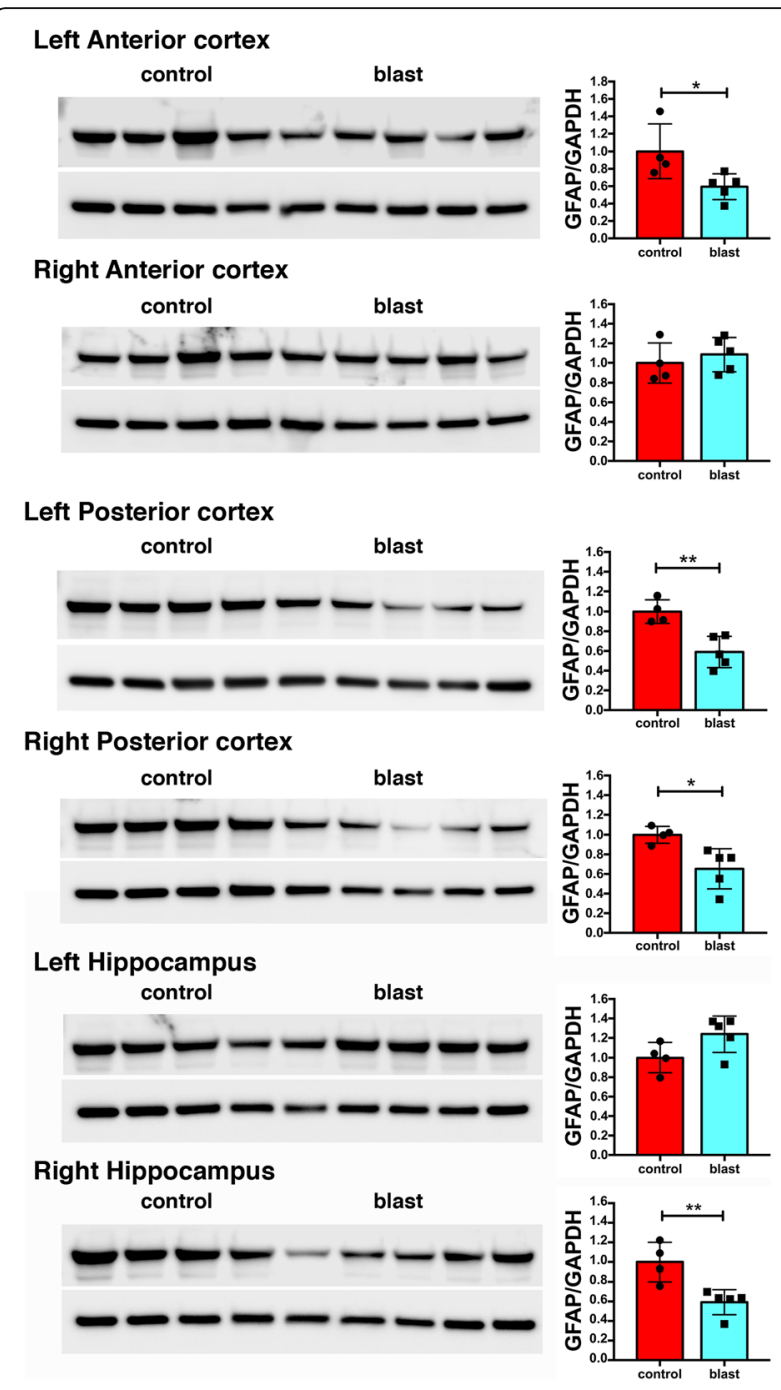

Fig. 6 GFAP levels in whole brain extracts following low-energy blast exposure. Immunoblotting for GFAP in extracts of brain regions from four control and five blast-exposed rats. Levels of expression were normalized to GAPDH as shown in the bar graphs. All lanes were loaded with $50 \mathrm{\mu g}$ of protein and represent individual animals. Statistical differences were assessed with unpaired $t$-tests $\left({ }^{*} p<0.05\right.$ ** $p<0.01)$. All animals were euthanized 6 weeks after blast exposure

blot analysis of the isolated vascular fractions (the same samples shown in Fig. 5a) with antibodies against NFH, $\alpha-I N X$, and NFM. In each case as in the proteomic studies, the levels of neuronal IFs were decreased by about 3 -fold in blast-exposed rats.

The presence of neuronal IFs in isolated vascular fractions suggested that neuronal fibers remain attached or at least tightly associated with purified brain vascular preparations. Immunostaining of vascular fractions for NFH showed focal staining sometimes with fine processes apparent, consistent with neuronal attachments to blood vessels (Fig. 7b). Our results are in agreement with others who have also observed neuronal IFs in highly purified preparations of mouse brain vasculature $[10,20]$. Their decrease following blast injury suggests that, like gliovascular, neurovascular attachments are being disrupted as a consequence of blast injury.

\section{Astroglial coverage of blood vessels in brain at 6 weeks after blast exposure}

The loss of gliovascular attachments in isolated vascular fractions would predict less astrocyte coverage of blood vessels in intact brain. To estimate astroglial coverage of blood vessels in brain, sections of control and blast-exposed animals were initially analyzed by GFAP and collagen IV immunohistochemical staining. Figures 8 and 9 show representative sections of the prelimbic cortex from five blast-exposed and five control animals euthanized at 6 weeks after blast exposure. No obvious differences were seen in any brain region examined between blast-exposed and control specimens.

While others have quantified astroglial coverage of human microvessels in brain by assessing the colocalization of GFAP and collagen IV immunostaining [6, 70], we found that this technique was not directly applicable to rat brain since vascular segments greatly enriched with GFAP immunoreactivity were very often present in areas devoid of collagen IV immunoreactivity (Figs. 8 and 9). This was observed in sections from both controls and blast-exposed rats, even when the tissue was subjected to a protease antigen retrieval protocol utilizing pepsin to unmask vascular collagen IV immunostaining [30, 34].

Although it failed to provide direct evidence of altered gliovascular coverage in brain, collagen IV immunostaining frequently revealed other vascular alterations induced by blast exposure. Figure 10 shows examples of large penetrating vessels in the hippocampal lacunosum moleculare where collagen IV immunoreactivity revealed lumens that were irregular with a thickened and multilayered appearance, which were at times collapsed (Fig. 10b-d). Figure 10f also shows an example of a penetrating cortical vessel in which the external layers of the adventitia have separated giving the vessel a double-barreled appearance.

In addition, perivascular GFAP-containing processes often appeared disturbed. Figure 11 shows blood vessels in the lacunosum moleculare of the hippocampus immunostained for $\alpha$-SMA and GFAP. Besides disturbed vascular smooth muscle ( $\alpha$-SMA), which often appeared irregular and vacuolated (Fig. 11b), intraluminal astrocytic processes were frequently visible (Fig. 11b-d). Figure $11 \mathrm{~d}$ in particular shows a parenchymal vessel that exhibits a large nest of intraluminal GFAP-immunoreactive processes. Similarly, abnormal $\alpha$-SMA immunostaining and intravascular GFAP-immunolabled processes were found in the brains of animals 10 months after blast exposure (Fig. 12). 

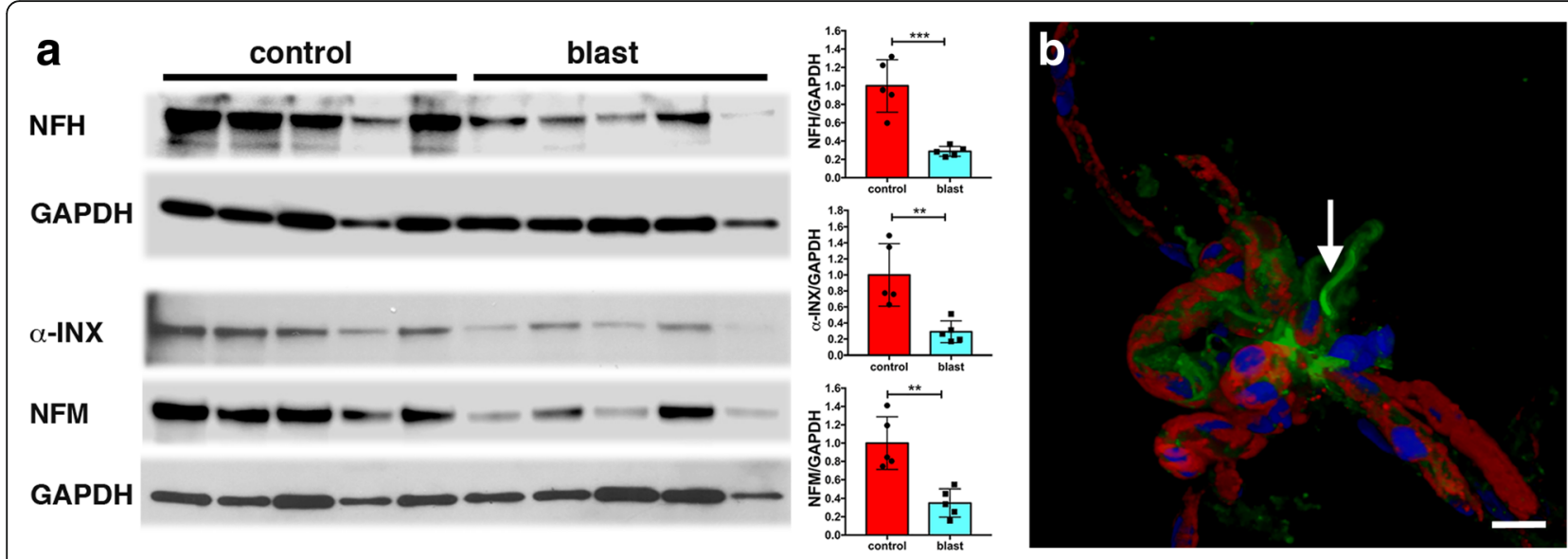

Fig. 7 Disruption of neurovascular interactions by low-energy blast exposures. Isolated brain vascular fractions were prepared from five control and five blast-exposed rats ( 6 weeks after blast exposure, same samples as shown in Fig. 5a). a Western blot analysis of the expression of NFH, a-INX and NFM in isolated vascular fractions. The top GAPDH panel represents the loading control for the NFH blot; the bottom GAPDH panel is the loading control for the a-INX and NFM blots. Blots were obtained by sequential probing of the same membrane. All lanes were loaded with $10 \mu \mathrm{g}$ of protein and contain protein from individual animals. Quantitation is shown in the right panels with expression normalized to GAPDH. Statistical differences were assessed with unpaired $t$-tests ( ${ }^{* *} p<0.01,{ }^{* * *} p<0.001, n=5 /$ group). b Isolated large cerebral vessel, likely a pial or penetrating arteriole, stained for NFH (green) and Griffonia simplicifolia isolectin B4 (red). Nuclei were stained with DAPI (blue). An arrow indicates an NFH-immunostained process that remains attached to the vessel. Other patches of focal NFH immunoreactivity are also visible. Scale bar, $20 \mu m$

\section{Degeneration of astrocytic endfeet in blast-injured brain} at 6 weeks following blast exposure revealed by EM

To gain a better understanding of how blast affects gliovascular connections at the ultrastructural level we examined sections from the motor cortex of rats harvested at 6 weeks after the last blast exposure. Figure 13 shows examples of small arterioles from control and blast-exposed rats.
Compared to the controls, the lumens of the blast-exposed vessels appeared irregular and thickened. In addition, compared to the tight astrocytic endfeet surrounding control vessels, perivascular astrocytic endfeet in the blast-exposed vessels were swollen and contained degenerating organelles. The lumens of the associated vessels were often irregular and collapsed. While the level of endfoot degeneration

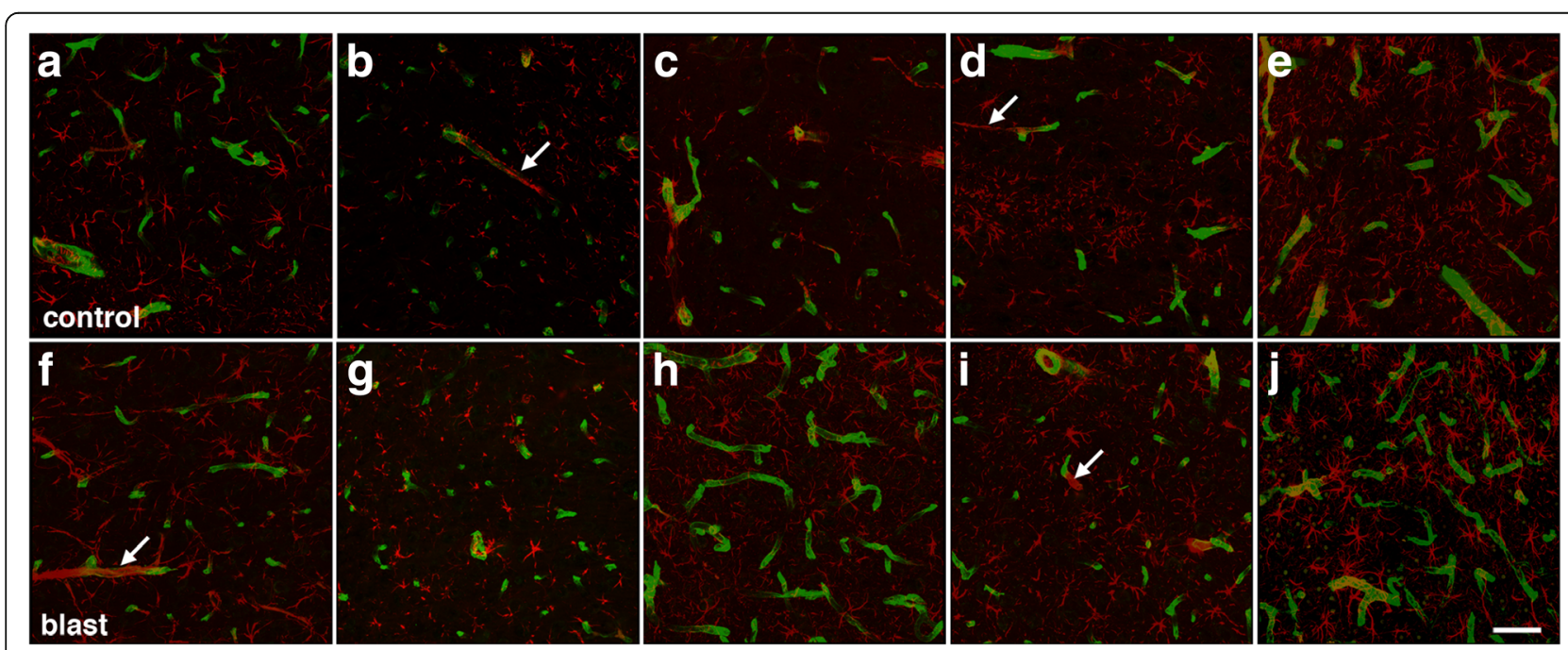

Fig. 8 Visualization of astrocyte coverage of the brain vasculature in control and blast-exposed rats. Sections from five individual control and five blast-exposed animals were immunostained for collagen IV (green) and GFAP (red) utilizing an antigen retrieval protocol employing pepsin treatment [30, 34]. Rats were euthanized 6 weeks after blast exposure. a-e Representative images from the prelimbic cortex from each individual control brain. $\mathbf{f}$-j Representative images from the prelimbic cortex from each individual blast exposed brain. Arrows in panels $(\mathbf{b}, \mathbf{d}, \mathbf{f})$ and $(\mathbf{i})$ indicate examples of perivascular astroglial fibers associated with domains on blood vessels that stained poorly with antibodies against collagen IV even after pepsin treatment. Scale bar, $40 \mu \mathrm{m}$ 


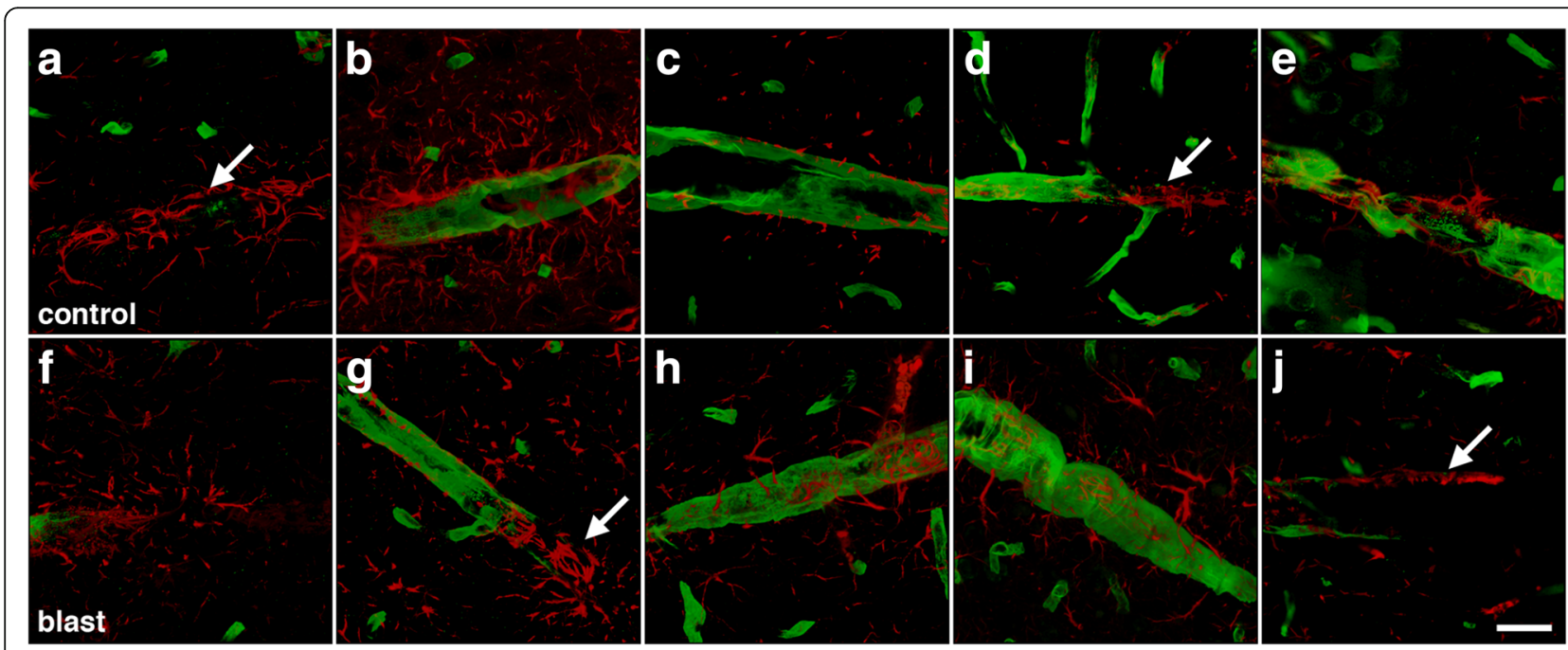

Fig. 9 Astrocyte coverage of large and medium sized parenchymal vessels in control and blast-exposed animals euthanized 6 weeks after exposure. Brain sections of five control and five blast-exposed animals were immunostained for collagen IV (green) and GFAP (red). a-e Representative images in the prelimbic cortex from each individual control animal. $\mathbf{f}$-j Representative images in the prelimbic cortex from each individual blast-exposed animal. Arrows in panels $(\mathbf{a}, \mathbf{d}, \mathbf{g})$ and $(\mathbf{j})$ indicate areas of perivascular GFAP expression in regions of blood vessels that stain poorly for collagen IV. Scale bar, $20 \mu \mathrm{m}$

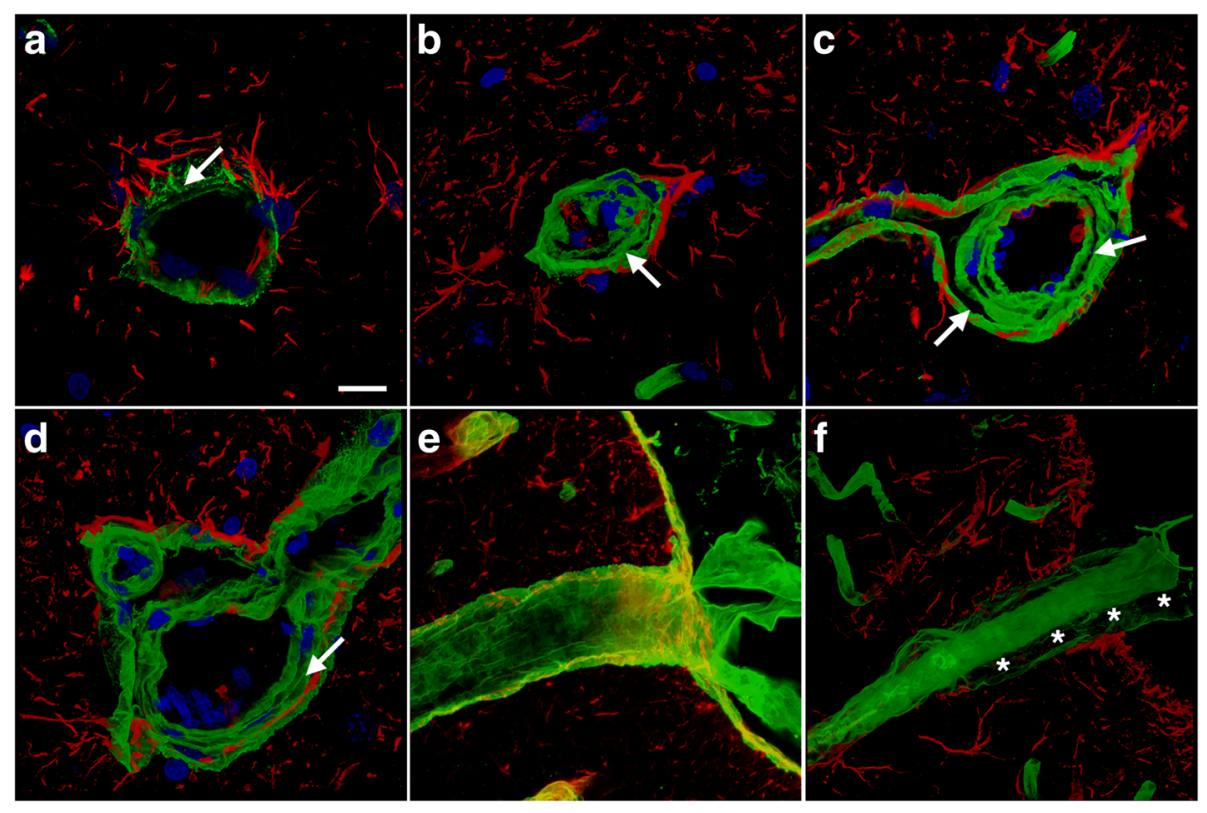

Fig. 10 Altered vascular extracellular matrix in blast-exposed animals. Brain sections of rats euthanized 6 weeks after blast exposure were immunolabeled for collagen IV (green) and GFAP (red) with DAPI nuclear staining (blue). Arrows in the panels indicate the collagen IV-rich layers, which include the endothelial basal membrane and adventitia. a-d Representative sections from the hippocampal stratum lacunosum moleculare from a control (a) and a blast-exposed rat (b-d). Note the separation of the collagen IV-rich layers in panels (b-d), resulting in a multilayered appearance of the collagen IV- immunostained extracellular matrix. In panel (b) the loss of structure in the collagen IV-rich layers resulted in collapse of the lumen. e-f Penetrating cortical vessels from control (e) or blast-injured (f) rats. The blast-exposed vessel in panel (f) exhibits a double-barreled appearance. Asterisks $\left(^{*}\right)$ in panel $(\mathbf{f})$ mark the separation of the adventitial layer from the tunica media of the blood vessel. Scale bar, $20 \mu \mathrm{m}$ 

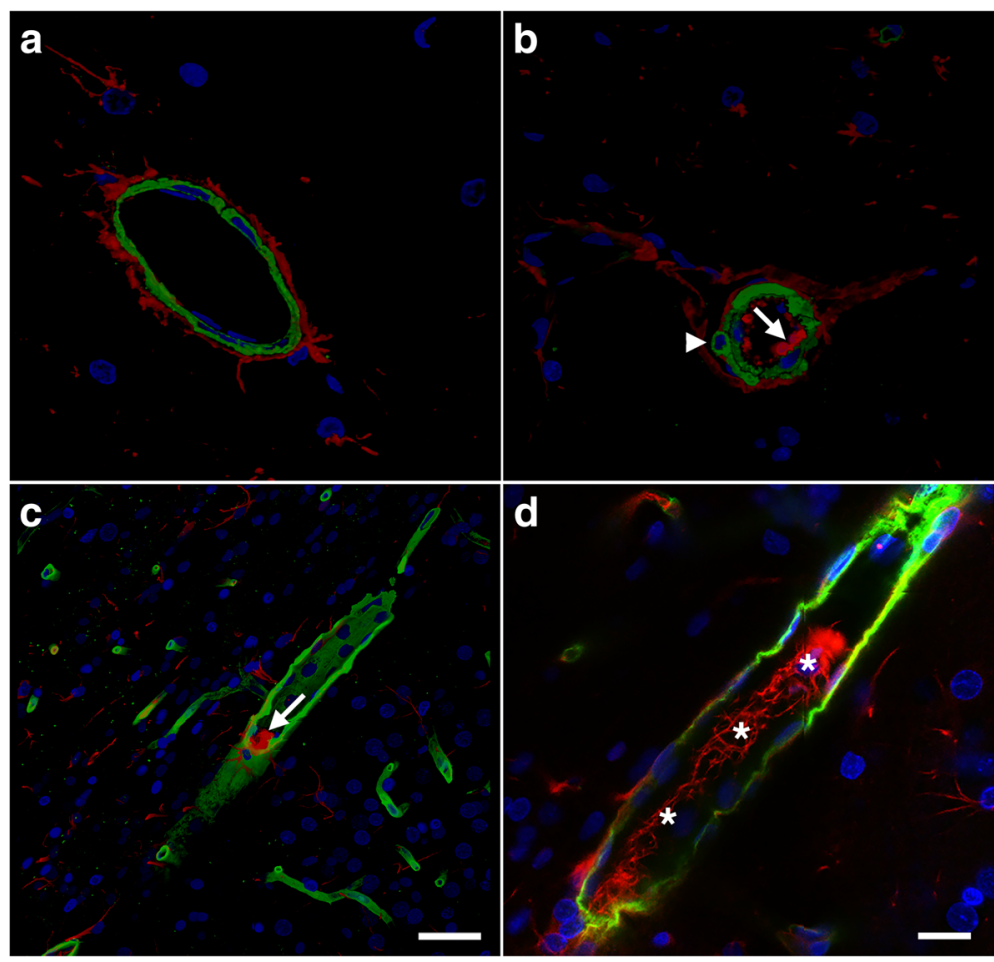

Fig. 11 Intraluminal astrocytic processes after blast exposure. Brain sections from rats sacrificed 6 weeks post-blast exposure were immunostained for GFAP (red) and a-SMA (green) with a DAPI nuclear counterstain (blue). a-d Representative sections of the hippocampal stratum lacunosum moleculare from a control (a) or a blast-exposed (b) rat. The arrow in panel (b) indicates the presence of intraluminal GFAP. The arrowhead in panel (b) indicates vacuolation in the smooth muscle (a-SMA staining). The smooth muscle layer appears thick, irregular and disorganized compared to that in the control in panel (a). c Panoramic 3D reconstruction of a large parenchymal vessel in a blast-exposed animal exhibiting intraluminal GFAP expression (arrow). d A $0.56-\mu m$-thick confocal optical section from the cell in panel (c) showing directionally oriented GFAPimmunostained processes (asterisks) within the lumen. Scale bars: $50 \mu \mathrm{m}$ for (a-c), $20 \mu \mathrm{m}$ for (d)

varied considerably, a count of 50 randomly sampled vessels in the blast-exposed specimen revealed that 9 (18\%) had clearly swollen astrocytic endfeet such as those illustrated in Fig. 13. Blast-exposed capillaries showed similar changes. Astrocytic changes were more obvious in blood vessels showing the most endothelial cell damage. Such changes were not observed in microvessels of controls.

\section{Blast-induced vascular occlusion by CD34-immunoreactive} cells in rats sacrificed 6 weeks after blast exposure

Some cerebral small vessels from blast-exposed animals also appeared to be occluded by CD34-expressing cells. Figure 14 shows sections double-immunostained for $\alpha$-SMA and CD34. Unlike controls in which CD34 immunostaining was confined to the adventitia, in blast-exposed animals CD34-immunoreactive elements were seen occluding the vessel lumen. Occlusions could also be identified by EM in blast-exposed rats (Fig. 14f). As in Figs. 11 and 12, the $\alpha$-SMA immunoreactivity in the blast-exposed microvessels shown in Fig. 14 appeared thickened, irregular and vacuolated, indicative of altered smooth muscle layers.
Recovery of GFAP and neuronal IF expression in isolated brain vascular fractions from blast-exposed rats 8 months after exposure

To determine whether GFAP and neuronal IF expression remained chronically decreased in isolated brain vascular fractions following blast injury, we studied a group of blast-exposed and control rats 8 months following blast injury. As shown in Fig. 15, GFAP and NFH levels were unchanged in the blast-exposed compared to control samples.

\section{Chronic blast-induced cerebral vascular pathology at 10} months following blast exposure revealed by micro-CT To determine whether blast induces chronic vascular pathology, we obtained high-resolution X-ray micro-CT scans of the brain vasculature of rats at 10 months after blast exposure following perfusion with the Brite $\mathrm{Vu}$ contrast agent. Figure 16 shows maximum intensity projection (MIP) images of the volume-rendered brain vasculature from the brains of 2 control and 2 blast-exposed animals reconstructed with Bruker's CTVox $3 \mathrm{D}$ visualization software. A generalized thinning of the cerebral vasculature is noticeable in the blast-exposed 


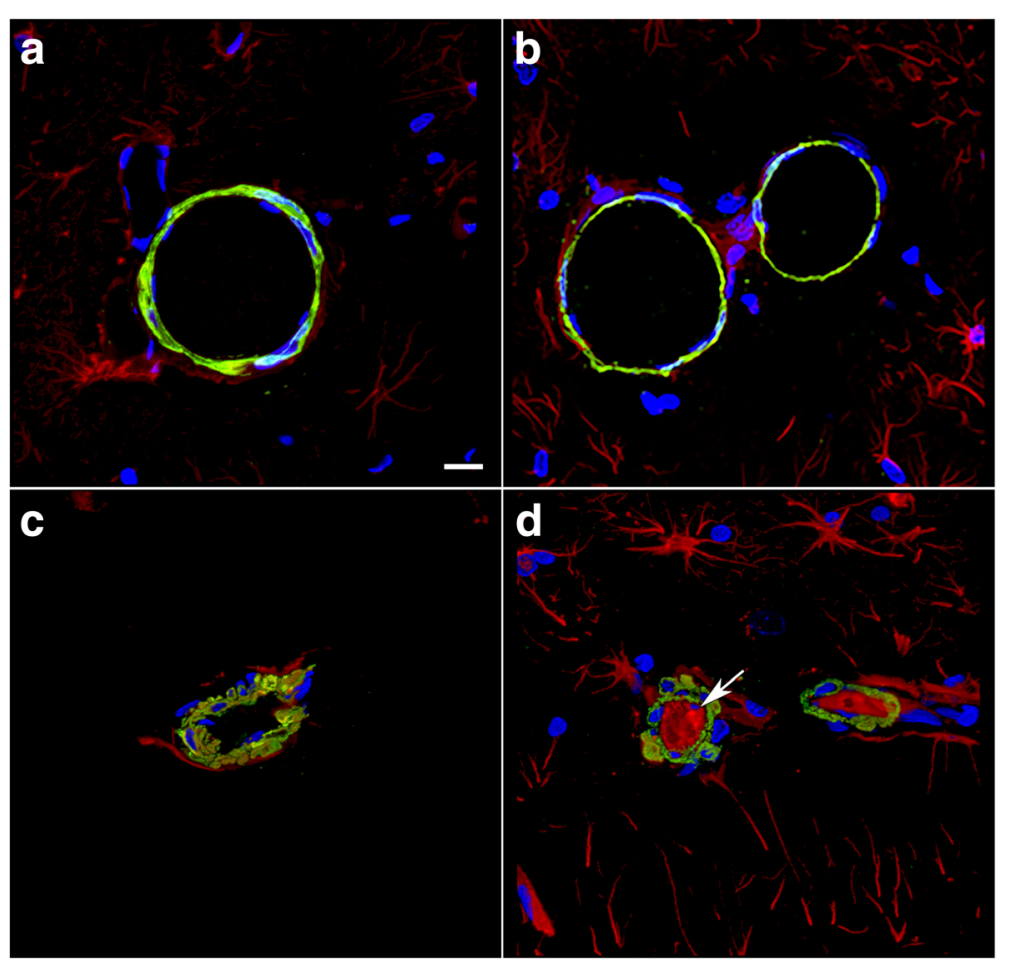

Fig. 12 Disrupted smooth muscle layers and intraluminal astrocytic processes are present in vessels 10 months after blast. Brain sections from rats sacrificed 10 months after the last blast exposure were immunostained for GFAP (red) and a-SMA (green) with a DAPI nuclear counterstain (blue). a-d Representative sections of the hippocampal stratum lacunosum moleculare from control (a-b) or blast-exposed (c-d) rats. The arrow in (d) indicates the presence of intraluminal GFAP. Note the vacuolation in the smooth muscle (a-SMA staining). Scale bar, $50 \mu \mathrm{m}$

animals (Fig. 16a-d). Panels e-p in Fig. 16 show visualization of the micro-CT data reconstructed for quantitation with the software Vesselucida. Quantitative analyses of these data showed a blast-induced decrease in total brain vascular length (about 50\%; $6.22 \times 10^{6} \pm 1.31 \times$ $10^{6} \mu \mathrm{m}$ for control vs $3.16 \times 10^{6} \pm 2.06 \times 10^{4} \mu \mathrm{m}$ for blast), total surface area (over 50\%; $9.99 \times 10^{8} \pm 1.55 \times 10^{8} \mu \mathrm{m}^{2}$ for control vs $4.68 \times 10^{8} \pm 1.88 \times 10^{7} \mu \mathrm{m}^{2}$ for blast) and total volume (almost $60 \% ; 1.52 \times 10^{10} \pm 1.17 \times 10^{9} \mu \mathrm{m}^{3}$ for control vs $0.62 \times 10^{10} \pm 5.17 \times 10^{8} \mu \mathrm{m}^{3}$ ). Imaging analyses also revealed a general loss of vascular organization with disruption of the regular radial patterns visible in control brain (Fig. 14i-j). Thus, despite recovery of GFAP and $\mathrm{NFH}$ associated with the vascular fraction, a chronic blast-induced vascular pathology remained as evidenced by micro-CT scanning, a finding consistent with prior work demonstrating chronic vascular pathology in this model between 6 and 10 months following blast exposure [31].

\section{Discussion}

We have been studying a rat model developed to mimic blast exposures that would be associated with human mTBI or a subclinical exposure, an exposure that we refer to as "low-level" [29]. In multiple studies the lack of generalized neuronal pathology at the light and EM levels has been confirmed as well as the absence of any generalized reactive astrocytosis or inflammatory reaction [2, 22, 26, 31-33]. Because multiple blast exposures have been common among veterans returning from Iraq and Afghanistan [28], for most studies including the present we used a design in which rats received three exposures delivered one per day on 3 consecutive days. Rats exposed to these blast conditions develop a variety of chronic PTSD-related behavioral traits [26, 64-67].

Despite the generally benign appearance of the brain in standard histopathology at low magnification, at the EM level, a prominent vascular pathology was visible [31]. To explore blast-induced molecular changes in the vasculature we conducted proteomic studies using purified brain vascular fractions. The present study stems from the unexpected observation that GFAP was decreased in isolated brain vascular fractions from blast-exposed animals (6 weeks after exposure). These findings were confirmed by quantitative Western blotting on a separate cohort of animals harvested also 6 weeks post-blast exposure.

Many studies have documented astrogliosis following higher level blast exposures [27], and a distinct type of glial scar has been described in humans exposed to blast 


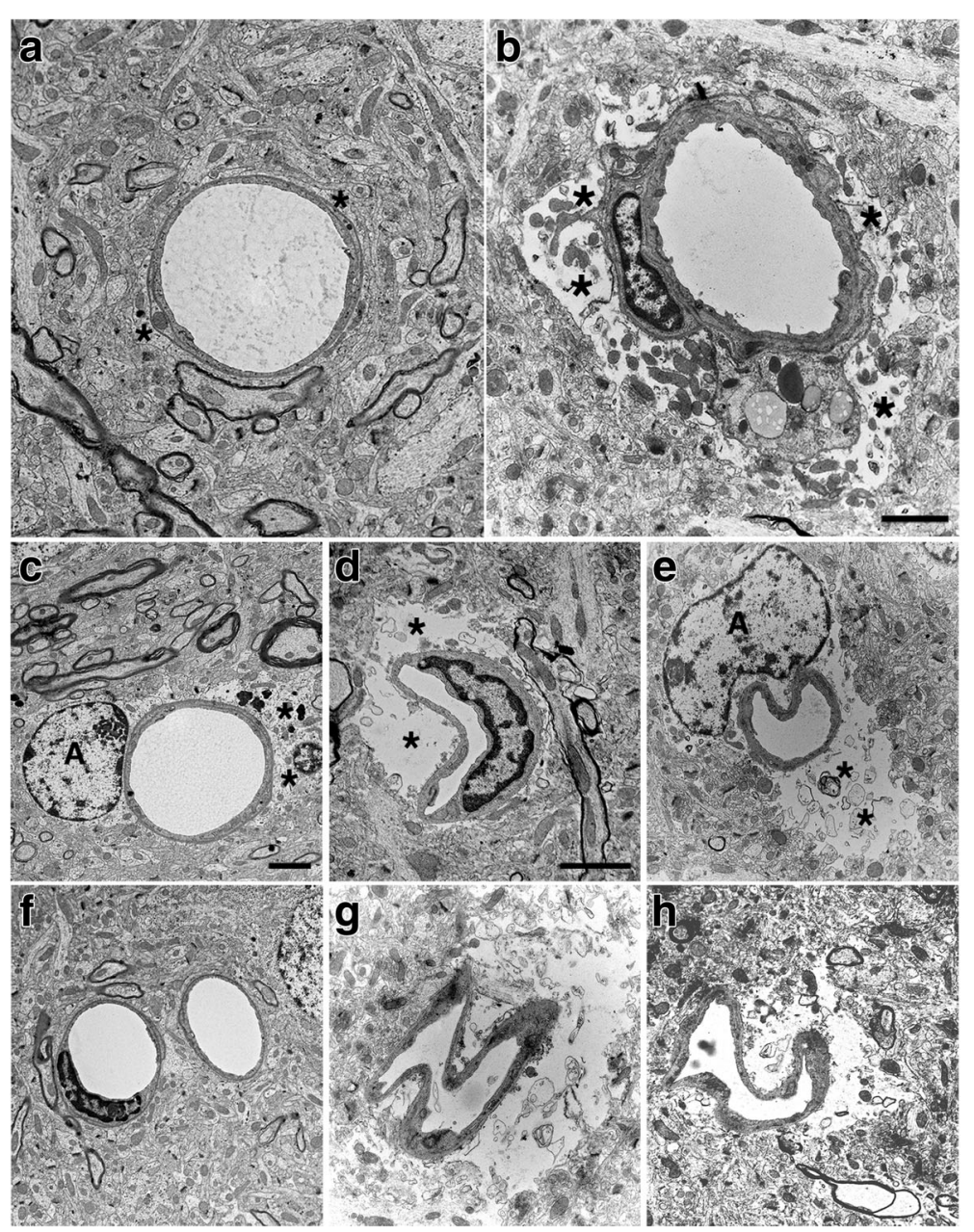

Fig. 13 Blast induced swelling and degeneration of astrocytic endfeet in the frontal motor cortex. a-f Electron micrographs are shown from sections of the frontal motor cortex of control $(\mathbf{a}, \mathbf{c}, \mathbf{f})$ and blast-injured $(\mathbf{b}, \mathbf{d}, \mathbf{e}, \mathbf{g}, \mathbf{h})$ rats. Animals were euthanized 6 weeks following blast exposure. Asterisks (*) indicate astrocytic endfeet, which are swollen and contain degenerating organelles in all the blast-exposed microvessels. The lumens of the microvessels in the blast-exposed animals also appear irregular and collapsed. Perivascular astrocytes (A) are visible in panels (c) and (e). Scale bars, $2 \mu \mathrm{m}$. Scale bar in panel (b) applies to panels (a) and (b). Scale bar in panel (c) applies to panels (c, e, f, g) and (h). Scale bar in panel (d) applies only to panel (d)

injury [79]. By contrast we found a decrease of GFAP in isolated brain vascular fractions from blast-exposed animals. In a regional analysis, GFAP was either normal or decreased in selected brain regions further arguing against any generalized astrogliosis in this model. Differences in GFAP in isolated vascular fractions did not seem to be accounted for by differences in the relative arterial vs. venous compositions of the vascular fractions isolated from control and blast-exposed brains as no differences were noted in Western blotting with antibodies against a preferential marker of arterial smooth muscle $(\alpha-S M A)$ or a marker of venous endothelial cells (EPHB4) [24]. Micro CT findings suggested a thinning of the brain vasculature following blast exposure. Because in the 2D-DIGE and Western immunoblotting analyses equal amounts of protein from blast-exposed and control vascular preparations were compared, the reduction in GFAP and neurofilament proteins cannot be explained by a loss of brain vasculature in blast-exposed animals. Loss of GFAP in isolated vascular fractions suggested a widespread loss of gliovascular connections following blast, a speculation confirmed with GFAP immunostaining of isolated vascular fractions. Loss of gliovascular coverage was not clearly visible in sections immunostained for GFAP and collagen IV. However, EM studies revealed swollen and disrupted astrocytic endfeet around small cortical vessels, suggesting that reduced GFAP levels previously observed in isolated vascular fractions may have resulted from loosened gliovascular connections (due to astrocytic degeneration) that were lost during the isolation procedure. Equally surprising in the initial proteomic study was a loss of a 

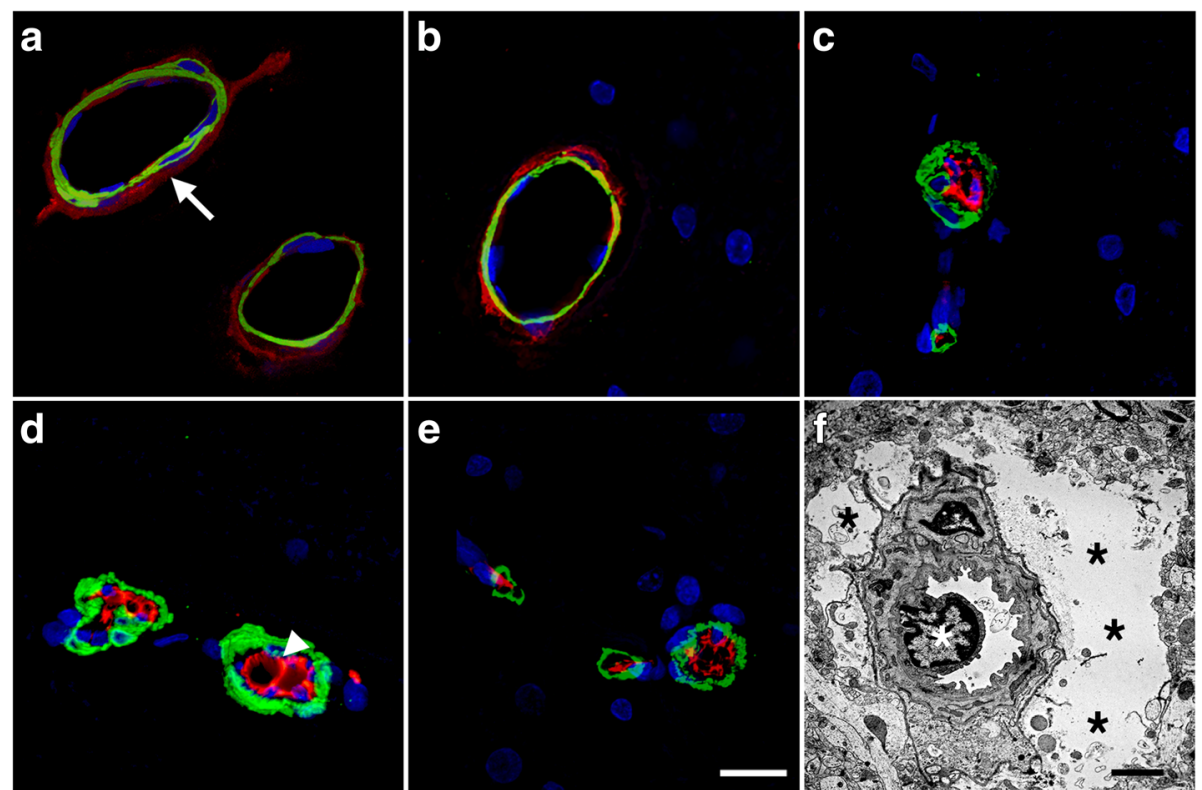

Fig. 14 Vascular smooth muscle alterations and arteriolar occlusion by CD34-expressing cells. a-e Brain sections from control and blast exposed rats sacrificed 6 weeks after blast exposure were immunostained with antibodies against vascular a-SMA (green) and CD34 (red). Nuclei were counterstained with DAPI (blue). Representative sections of the hippocampal stratum lacunosum moleculare from control (a-b) and blast-exposed (c-e) rats. Note that CD34 immunoreactivity is present in the adventitia of control vessels (arrow in a) but is absent in the blast-injured vessels. The smooth muscle layer appears thickened and irregular in the blast-exposed vessels, and CD34-expressing cells are present inside the vessel lumen (arrow head in $\mathbf{d}$ ). $\mathbf{f}$ Electron micrograph of a cortical arteriole from the frontal cortex of a blast-exposed rat showing a cellular occlusion (white asterisk) similar to those shown in panels $(\mathbf{c}, \mathbf{d})$ and $(\mathbf{e})$. Black asterisks $\left(^{*}\right)$ in panel (f) mark swollen astrocytic endfeet. Scale bars: $20 \mu m$ for $(\mathbf{a}-\mathbf{e}), 2 \mu \mathrm{m}$ for $(\mathbf{f})$

series of neuronal IF proteins which suggested widespread loss of neurovascular connections as well.

Although loss of GFAP and neuronal IF proteins associated with the vasculature was reversed by 8 months after blast exposure, a chronic vascular pathology was still visible histologically and by micro-CT scanning. These findings suggest that normalization of GFAP and IF protein levels in isolated vascular fractions, while likely signaling the tightening of gliovascular and neurovascular connections, does not translate into a resolution of the vascular pathology. Chronic vascular alterations induced by blast exposures have been previously documented in this model at 6-10 months following blast exposure [31].

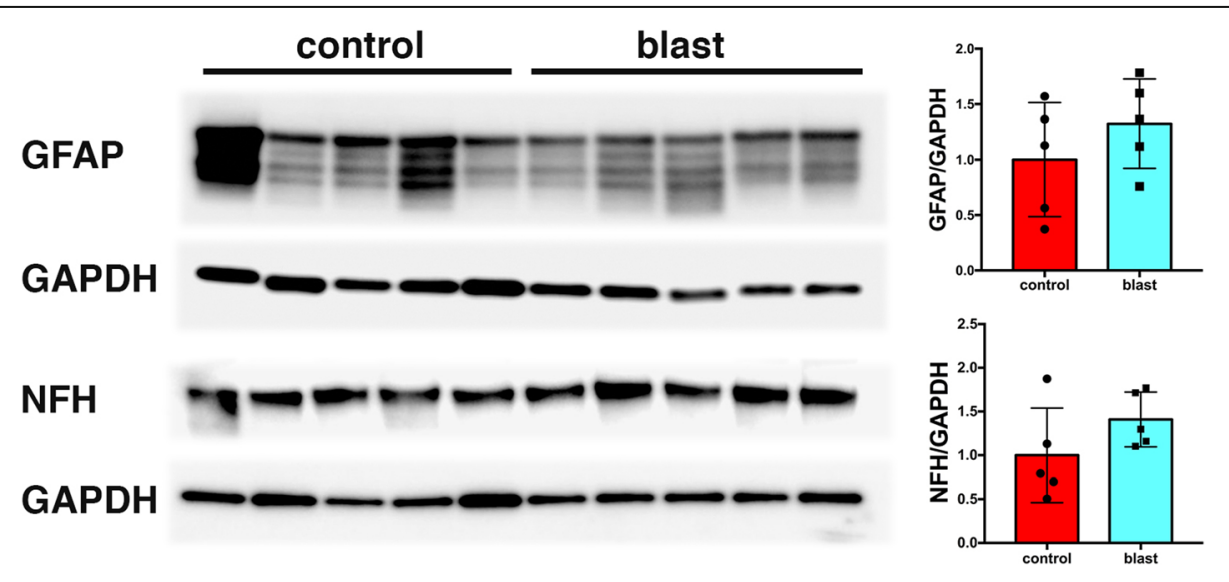

Fig. 15 GFAP and NFH levels in blast injured vascular fractions recover with time following blast injury. Brain vascular fractions were isolated from five control and five blast-exposed rats 8 months after the last blast exposure. The figure shows immunoblotting for GFAP and NFH followed by reprobing for GAPDH. All lanes were loaded with $10 \mu \mathrm{g}$ of protein and contain protein from individual animals. Quantification with expression normalized to GAPDH is shown on the right. No significant differences were observed between blast-exposed and control animals 


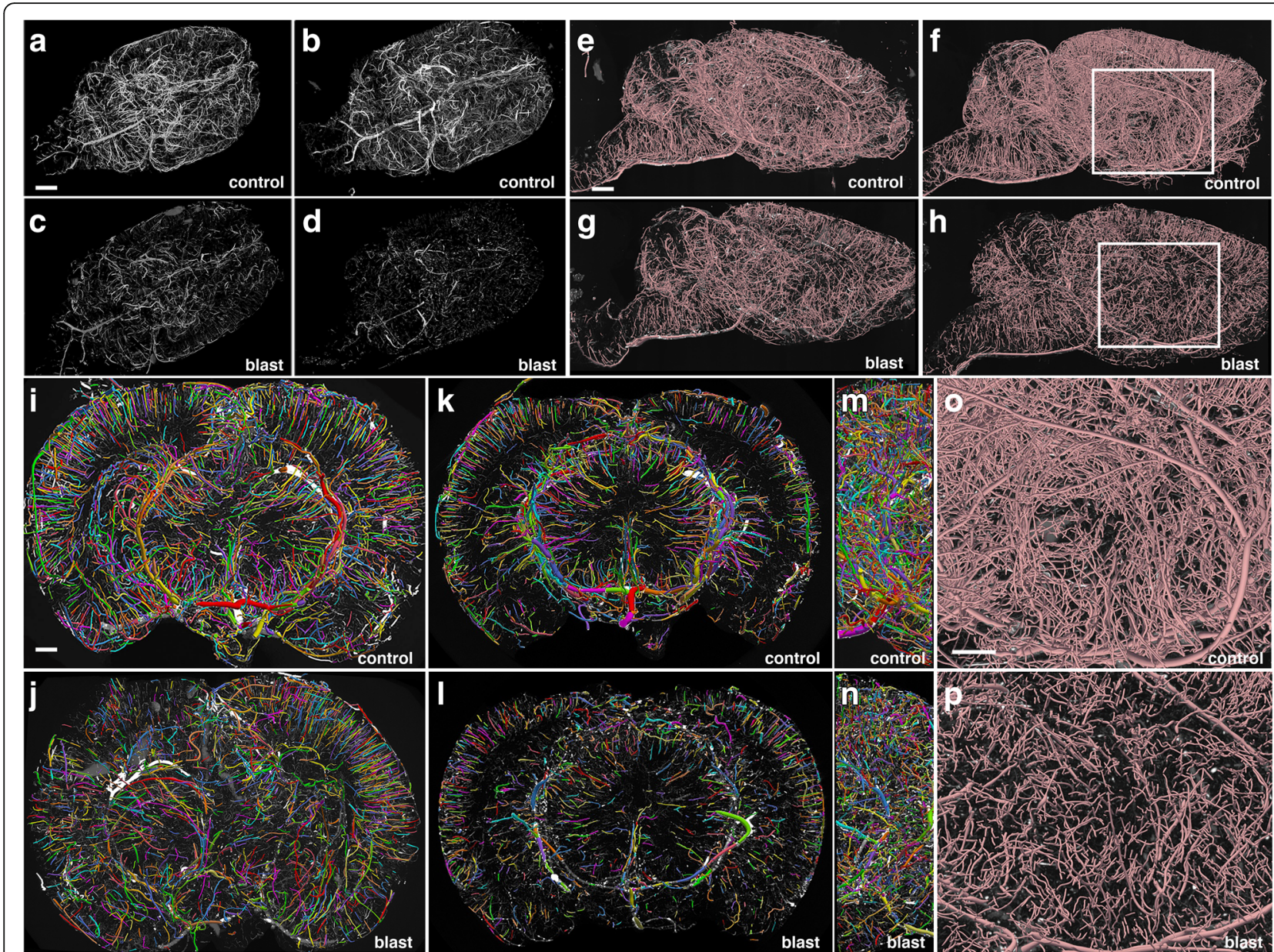

Fig. 16 Chronic vascular pathology in blast-exposed rats revealed by micro-CT scanning. Two control and two blast-exposed rats were transcardially perfused with the Brite Vu contrast agent at 10 months after blast exposure. Brains were scanned at a resolution of $7.5 \mu \mathrm{m}$ using equispaced angles of view around $360^{\circ}$, and 3D reconstructions were prepared with Bruker's CTVox 3D visualization software. a-d MIP images of volume-rendered brain vasculature from two control $(\mathbf{a}, \mathbf{b})$ and two blast-exposed $(\mathbf{c}, \mathbf{d})$ rats revealed diffuse thinning of the brain vasculature in the blast-exposed rats. Scale bar, $2 \mathrm{~mm}$. e-h Trace sagittal reconstructions used for the automated quantitation from control (e-f) and blastexposed rats ( $\mathbf{g}-\mathbf{h}) \mathbf{o}-\mathbf{p}$ Higher magnification views of the regions outlined by the boxes in panels $(\mathbf{f})$ and (h). Scale bars, $1 \mathrm{~mm}$ for $(\mathbf{e}-\mathbf{h})$, and 0.6 $\mathrm{mm}$ for (o-p). $\mathbf{i}-\mathbf{n}$ Reconstructions of coronal optical sections from the brains of control (i, $\mathbf{k})$ and blast-exposed (j, $\mathbf{l})$ animals. Panels (i) and (j) correspond approximately to coordinates interaural $12.24-9.48 \mathrm{~mm}$ and panels (k) and (I) correspond approximately to coordinates interaural 6.94-3.24 mm. Lateral views of (i) and (j) are shown in $(\mathbf{m})$ and $(\mathbf{n})$, respectively. Vessels were color coded to allow visualization of individual vessels automatically traced by the Vesselucida 360 software. Note the general loss of radial organization in the blast-exposed shown in panel (j). Scale bar, $1 \mathrm{~mm}$ for (i-n)

Vascular pathology is a well-described feature of blast-related brain injury in both animals and humans $[1,7,18,19,27,31,32,37,42,46-48,51-53,55,58$, $61,68,69,72,73,76,80,84,87]$ with multiple studies describing increases in BBB permeability [1, 36, $45,49,52,54,56,57,60,61,71,74,81,85,90,92$, 93]. What is novel about the present studies is the suggestion that the basis for these changes is at least in part the result of a widespread disruption of gliovascular and neurovascular connections in a model that lacks any obvious diffuse neuronal structural pathology. The fact that these changes were apparent in Western blots of vascular fractions obtained from whole brain further supports the widespread nature of the changes.

Several prior studies have described ultrastructural changes in astrocytic endfeet following blast exposure $[37,46,55]$. In a rat model of blast injury, Kaur et al. [46] observed that astrocytes in the cerebral and cerebellar cortex were hypertrophied with swollen endfeet that were sparsely populated with organelles. However, this pathology appeared only transiently and was absent in rats studed 14-28 days after blast exposure. Lu et al. [55] described hypertrophied and "watery" astrocytic endfeet in a non-human primate model utilizing live explosives. These changes however occurred in the context 
of a broader neuronal, glial and microglial pathology that was absent in our model. Goldstein et al. [37] described swollen astroyctic endfeet in a mouse model of blast injury. This pathology however also occured in the setting of a broader neuropathology with neuronal tau pathology, axonal changes and widespread astrogliosis. In addition in these studies when the head was immobilized hippocampal learning and memory deficits were no longer apparent arguing that effects were occurring primarily on the basis of acceleration-deceleration/rotational injury and not as a primary effect of blast, unlike in our studies in which the head was immobilized and no broader neuropathology was apparent.

Several limitations of this study must be mentioned. Firstly the time course of the progression of gliovascular and neurovascular changes has not been defined. Because of our interest in the aspects of blast exposure most relevant to the veteran population we have focused on blast's effects on the subacute (6 weeks) to chronic (8-10 month) time periods after exposure. In a previous study we described acute vascular pathology $24-72 \mathrm{~h}$ after blast exposure [31]. However, these studies will need to be expanded into a broader longitudinal study that will include ultrastructural analysis of samples from the acute to chronic phases. While the levels of vascular-associated GFAP recover in Western blots to control levels at 8 months post-exposure, we do not know whether this reflects recovery of normal gliovascular connections. In addition, the micro $\mathrm{CT}$ results further support the widespread nature and chronicity of the vascular degenerative processes in this model in a manner that is more easily appreciated than in tissue sections. However, it will be necessary to expand the sample size and time points for the micro CT studies in order to capture the full evolution of the disease process.

It should also be noted that we have not explored the full extent of the molecular changes in blast-exposed vessels. The 2D-DIGE system by its nature only detects differences in relatively high abundance proteins. There are likely many other changes that went undetected in our screen. Others have reported changes in the BBB tight-junction proteins occludin, claudin-5, and zonula occludens 1 (ZO-1) as well as in VE-cadherin, Chemokine (C-C Motif) Ligand 2 (CCL2) and VEGF following blast exposure $[1,3$, $54,57,75,89$ ] and to date we have only identified slightly more than a dozen of the 77 spots that differed between control and blast-exposed in the initial screen.

Because gliovascular and neurovascular interactions control cerebral blood flow at multiple levels, their disruption following blast exposure would be expected to affect cerebral autoregulation. Astrocytes play important roles in mediating neurovascular coupling (reviewed in [43]). Glutamate released from neurons acts on astrocytic metabotropic glutamate receptors to evoke $\mathrm{Ca}^{2}$
${ }^{+}$-dependent release of vasoactive metabolites from astrocytic endfeet. Under normoxic conditions, astrocytic $\mathrm{Ca}^{2+}$ signaling results in vasodilation, whereas under hyperoxia, vasoconstriction is favored.

Vascular radius is the most powerful determinant of blood flow, with even small changes in lumen diameter having significant effects on cerebral blood flow [63]. Cerebral pial vessels are innervated in the adventitial layer by perivascular nerves originating from both autonomic and sensory ganglia of sympathetic, parasympathetic and trigeminal origin (reviewed in [40]). Sympathetic terminals have significant vasoconstrictor effects in large cerebral arteries. Under conditions of elevated hydrostatic pressure, sympathetic vasoconstriction protects against increases in venous pressure, disruption of the BBB and edema formation. Stimulation of parasympathetic nerves has potent vasodilator effects on cerebral arteries, resulting in increased blood flow. Additional intrinsic cerebral hemodynamic regulatory mechanisms exist, as sympathetically and parasympathetically denervated animals exhibit cerebral blood flow autoregulation [11]. Thus altered neurovascular connections could disrupt cerebral autoregulation at multiple levels.

In addition to disrupted gliovascular and neurovascular signaling, the cerebral circulation could be also impaired by alterations in vascular smooth muscle. As demonstrated here, blast exposure results in structural alterations of the arterial medial smooth muscle layer. Interestingly, blast-induced vasospasm has been suggested to initiate a phenotypic switch in vascular smooth muscle cells that causes long-term vascular remodeling $[4,39]$. Whether the changes observed here are part of such a switch will require further study.

Moreover, in some small cortical and hippocampal arterial vessels, lumenal occlusion by cells expressing CD34 (and sometimes GFAP) was observed along with depletion of the $\mathrm{CD}_{3} 4^{+}$cells in the adventitia. CD34-expressing cells are a well-characterized population of stem cells that have the capacity to reconstitute the hematopoietic system [59]. In the arterial adventitia, these cells are likely remnants of earlier developmental stages and may participate in repair processes, as they are capable of differentiating into different vascular cell types, including smooth muscle cells, pericyte-like cells, CD34-expressing angiogenic progenitors and macrophages [62, 82]. The concomitant depletion of arterial adventitial CD34-expressing cells together with the partial arterial occlusion they cause argues for the migration of these cells from the adventitia into the lumen as a step possibly related to vascular repair mechanisms.

\section{Conclusion}

Our results show that blast exposure affects cellular interactions within the neurovascular unit including loss of gliovascular and neurovascular interactions as well as 
smooth muscle degeneration. The cognitive and other behavioral alterations that develop following blast exposure in both humans and animal models $[25,26,28,29$, 64-66] may in turn be caused by defective circulatory effects resulting from disrupted neurogliovascular cellular communication.

\section{Acknowledgements}

We thank Dr. Virginia Lee for generously providing the rat anti-GFAP antibody. We thank Timothy Tetreault for assistance with automatic quantification of vasculature. Work described in this paper was supported by the Department of Veterans Affairs, Veterans Health Administration, Rehabilitation Research and Development Service Awards 1 101RX000996, 1 I01 RX000684, 1 121 RX002069-01 and 1 I21 RX002876-01; by Department of Defense work unit number 0000B999.0000.000.A1503 and by NIH grant P50 AG005138. PL was a Carolyn L. Kuckein Student Research Fellow of the Alpha Omega Alpha Honor Medical Society. MAGS, RDG, GMP, GE, AET, SG and STA are employees of the U.S. government. This work was prepared as part of their official duties. Title 17 U.S.C. \$105 provides that 'Copyright protection under this title is not available for any work of the United States Government.' Title 17 U.S.C. §101 defines a U.S. Government work as a work prepared by a military service member or employee of the U.S. Government as part of that person's official duties. The views expressed in this article are those of the authors and do not necessarily reflect the official policy or position of the Department of the Navy, Department of Defense, Department of Veterans Affairs, nor the U.S. Government.

\section{Authors' contributions}

MAGS, RDG, GPG, SG, WGJ, SG, SJT, PRH and GAE: design of experiments, analysis and interpretation of data, design of neuropathological characterization, biochemical assays and manuscript writing; GMP, CS, DV and AS: execution of neuropathological characterization; PL and WGJ: electron microscopy; RWH, BA, RM and MAGS: micro-CT scanning; SJT: morphological and automated quantitative profiling of the cerebral vasculature; STA, RMM and AES: design and conduct of blast experiments, interpretation of data and manuscript writing. All authors read and approved the manuscript.

\section{Ethics approval}

All studies involving animals were reviewed and approved by the Institutional Animal Care and Use Committees of the Walter Reed Army Institute of Research (WRAIR) Naval Medical Research Center and the James J. Peters VA Medical Center.

\section{Competing interests}

SJT is the Scientific Director at MBF Bioscience.

\section{Publisher's Note}

Springer Nature remains neutral with regard to jurisdictional claims in published maps and institutional affiliations.

\footnotetext{
Author details

${ }^{1}$ General Medical Research Service, James J. Peters Department of Veterans Affairs Medical Center, 130 West Kingsbridge Road, Bronx, NY 10468, USA. ${ }^{2}$ Department of Psychiatry, Icahn School of Medicine at Mount Sinai, One Gustave Levy Place, New York, NY 10029, USA. ${ }^{3}$ Friedman Brain Institute, Icahn School of Medicine at Mount Sinai, New York, NY 10029, USA. ${ }^{4}$ Research and Development Service, James J. Peters Department of Veterans Affairs Medical Center, 130 West Kingsbridge Road, Bronx, NY 10468, USA. ${ }^{5}$ Department of Neurology, Icahn School of Medicine at Mount Sinai, One Gustave Levy Place, New York, NY 10029, USA. ${ }^{6}$ Fishberg Department of Neuroscience, Icahn School of Medicine at Mount Sinai, New York, NY 10029, USA. ${ }^{7}$ Department of Neurotrauma, Operational and Undersea Medicine Directorate, Naval Medical Research Center, 503 Robert Grant Avenue, Silver Spring, MD 20910, USA. ${ }^{8}$ Department of Surgery, Uniformed Services University of the Health Sciences, Bethesda, MD 20914, USA. ${ }^{9}$ Micro Photonics, Inc., 1550 Pond Road, Allentown, PA 18104, USA. ${ }^{10}$ MBF Bioscience, 185 Allen Brook Lane, Williston, VT 05495, USA. ${ }^{11}$ Department of Genetics and Genomic Sciences, Icahn School of Medicine at Mount Sinai, New York, NY 10029, USA. ${ }^{12}$ Mount Sinai Alzheimer's Disease Research Center and the Ronald M. Loeb Center for Alzheimer's Disease, Icahn School
}

of Medicine at Mount Sinai, New York, NY 10029, USA. ${ }^{13}$ NFL Neurological Care Center, Icahn School of Medicine at Mount Sinai, New York, NY 10029, USA. ${ }^{14}$ Department of Geriatrics and Palliative Care, Icahn School of Medicine at Mount Sinai, New York, NY 10029, USA. ${ }^{15}$ Neurology Service, James J. Peters Department of Veterans Affairs Medical Center, 130 West Kingsbridge Road, Bronx, NY 10468, USA.

Received: 14 November 2018 Accepted: 6 December 2018

Published online: 09 January 2019

\section{References}

1. Abdul-Muneer PM, Schuetz H, Wang F, Skotak M, Jones J, Gorantla S, Zimmerman MC, Chandra N, Haorah J (2013) Induction of oxidative and nitrosative damage leads to cerebrovascular inflammation in an animal model of mild traumatic brain injury induced by primary blast. Free Radic Biol Med 60:282-291. https://doi.org/10.1016/j.freeradbiomed.2013.02.029

2. Ahlers ST, Vasserman-Stokes E, Shaughness MC, Hall AA, Shear DA, Chavko M, McCarron RM, Stone JR (2012) Assessment of the effects of acute and repeated exposure to blast overpressure in rodents: toward a greater understanding of blast and the potential ramifications for injury in humans exposed to blast. Front Neurol 3:32

3. Ahmed F, Plantman S, Cernak I, Agoston DV (2015) The temporal pattern of changes in serum biomarker levels reveals complex and dynamically changing pathologies after exposure to a single low-intensity blast in mice. Front Neurol 6:114. https://doi.org/10.3389/fneur.2015.00114

4. Alford PW, Dabiri BE, Goss JA, Hemphill MA, Brigham MD, Parker KK (2011) Blast-induced phenotypic switching in cerebral vasospasm. Proc Natl Acad Sci U S A 108:12705-12710. https://doi.org/10.1073/pnas.1105860108

5. Armonda RA, Bell RS, Vo AH, Ling G, DeGraba TJ, Crandall B, Ecklund J, Campbell WW (2006) Wartime traumatic cerebral vasospasm: recent review of combat casualties. Neurosurgery 59:1215-1225; discussion 1225. https:// doi.org/10.1227/01.NEU.0000249190.46033.94

6. Armulik A, Genove G, Mae M, Nisancioglu MH, Wallgard E, Niaudet C, He L, Norlin J, Lindblom P, Strittmatter K et al (2010) Pericytes regulate the bloodbrain barrier. Nature 468:557-561. https://doi.org/10.1038/nature09522

7. Balaban C, Jackson RL, Liu J, Gao W, Hoffer ME (2016) Intracranial venous injury, thrombosis and repair as hallmarks of mild blast traumatic brain injury in rats: lessons from histological and immunohistochemical studies of decalcified sectioned heads and correlative microarray analysis. J Neurosci Methods 272:56-68. https://doi.org/10.1016/j.jneumeth.2016.02.001

8. Bauman RA, Ling G, Tong L, Januszkiewicz A, Agoston D, Delanerolle N, Kim Y, Ritzel D, Bell R, Ecklund J et al (2009) An introductory characterization of a combat-casualty-care relevant swine model of closed head injury resulting from exposure to explosive blast. J Neurotrauma 26:841-860

9. Bir C, Vandevord P, Shen Y, Raza W, Haacke EM (2012) Effects of variable blast pressures on blood flow and oxygen saturation in rat brain as evidenced using MRI. Magn Reson Imaging 30:527-534. https://doi.org/10. 1016/j.mri.2011.12.003

10. Boulay AC, Saubamea B, Decleves X, Cohen-Salmon M (2015) Purification of Mouse Brain Vessels. J Vis Exp:e53208. https://doi.org/10.3791/53208

11. Busija DW, Heistad DD (1984) Factors involved in the physiological regulation of the cerebral circulation. Rev Physiol Biochem Pharmacol 101:161-211

12. Carr W, Stone JR, Walilko T, Young LA, Snook TL, Paggi ME, Tsao JW, Jankosky CJ, Parish RV, Ahlers ST (2016) Repeated low-level blast exposure: a descriptive human subjects study. Mil Med 181:28-39. https://doi.org/10. 7205/MILMED-D-15-00137

13. Cernak I (2010) The importance of systemic response in the pathobiology of blast-induced neurotrauma. Front Neurol 1:151

14. Chaudhry FA, Lehre KP, van Lookeren Campagne M, Ottersen OP, Danbolt NC, Storm-Mathisen J (1995) Glutamate transporters in glial plasma membranes: highly differentiated localizations revealed by quantitative ultrastructural immunocytochemistry. Neuron 15:711-720

15. Chavko M, Koller WA, Prusaczyk WK, McCarron RM (2007) Measurement of blast wave by a miniature fiber optic pressure transducer in the rat brain. J Neurosci Methods 159:277-281

16. Chavko M, Prusaczyk WK, McCarron RM (2006) Lung injury and recovery after exposure to blast overpressure. J Trauma 61:933-942

17. Chavko M, Watanabe T, Adeeb S, Lankasky J, Ahlers ST, McCarron RM (2011) Relationship between orientation to a blast and pressure wave propagation inside the rat brain. J Neurosci Methods 195:61-66 
18. Chen HJ, Xu C, Li Y, Chen ZQ, Li GH, Duan ZX, Li XX, Zhang JY, Wang Z, Feng $\mathrm{H}$ et al (2015) An open air research study of blast-induced traumatic brain injury to goats. Chin J Traumatol 18:267-274

19. Cheng J, Gu J, Ma Y, Yang T, Kuang Y, Li B, Kang J (2010) Development of a rat model for studying blast-induced traumatic brain injury. J Neurol Sci 294:23-28

20. Chun HB, Scott M, Niessen S, Hoover H, Baird A, Yates J 3rd, Torbett BE, Eliceiri BP (2011) The proteome of mouse brain microvessel membranes and basal lamina. J Cereb Blood Flow Metab 31:2267-2281. https://doi.org/ 10.1038/jcbfm.2011.104

21. Courtney AC, Courtney MW (2009) A thoracic mechanism of mild traumatic brain injury due to blast pressure waves. Med Hypotheses 72:76-83

22. De Gasperi R, Gama Sosa MA, Kim SH, Steele JW, Shaughness MC, MaudlinJeronimo E, Hall AA, Dekosky ST, McCarron RM, Nambiar MP et al (2012) Acute blast injury reduces brain abeta in two rodent species. Front Neurol 3:177. https://doi.org/10.3389/fneur.2012.00177

23. DeKosky ST, Ikonomovic MD, Gandy S (2010) Traumatic brain injury: football, warfare, and long-term effects. N Engl J Med 363:1293-1296

24. dela Paz NG, D'Amore PA (2009) Arterial versus venous endothelial cells. Cell Tissue Res 335:5-16. https://doi.org/10.1007/s00441-008-0706-5

25. Elder GA (2015) Update on TBI and cognitive impairment in military veterans. Curr Neurol Neurosci Rep 15:68. https://doi.org/10.1007/s11910015-0591-8

26. Elder GA, Dorr NP, De Gasperi R, Gama Sosa MA, Shaughness MC, Maudlin-Jeronimo E, Hall AA, McCarron RM, Ahlers ST (2012) Blast exposure induces post-traumatic stress disorder-related traits in a rat model of mild traumatic brain injury. J Neurotrauma 29:2564-2575. https://doi.org/10.1089/neu.2012.2510

27. Elder GA, Gama Sosa MA, De Gasperi R, Stone JR, Dickstein DL, Haghighi F, Hof PR, Ahlers ST (2015) Vascular and inflammatory factors in the pathophysiology of blast-induced brain injury. Front Neurol 6:48. https://doi. org/10.3389/fneur.2015.00048

28. Elder GA, Mitsis EM, Ahlers ST, Cristian A (2010) Blast-induced mild traumatic brain injury. Psychiatr Clin North Am 33:757-781

29. Elder GA, Stone JR, Ahlers ST (2014) Effects of low-level blast exposure on the nervous system: is there really a controversy? Front Neurol 5:269. https://doi.org/10.3389/fneur.2014.00269

30. Franciosi S, De Gasperi R, Dickstein DL, English DF, Rocher AB, Janssen WG, Christoffel D, Sosa MA, Hof PR, Buxbaum JD et al (2007) Pepsin pretreatment allows collagen IV immunostaining of blood vessels in adult mouse brain. J Neurosci Methods 163:76-82. https://doi.org/10.1016/j.jneumeth.2007.02.020

31. Gama Sosa MA, De Gasperi R, Janssen PL, Yuk FJ, Anazodo PC, Pricop PE, Paulino AJ, Wicinski B, Shaughness MC, Maudlin-Jeronimo E et al (2014) Selective vulnerability of the cerebral vasculature to blast injury in a rat model of mild traumatic brain injury. Acta Neuropathol Commun 2:67. https://doi.org/10.1186/2051-5960-2-67

32. Gama Sosa MA, De Gasperi R, Paulino AJ, Pricop PE, Shaughness MC, Maudlin-Jeronimo E, Hall AA, Janssen WG, Yuk FJ, Dorr NP et al (2013) Blast overpressure induces shear-related injuries in the brain of rats exposed to a mild traumatic brain injury. Acta Neuropathol Commun 1:51. https://doi.org/ 10.1186/2051-5960-1-51

33. Gama Sosa MA, De Gasperi R, Perez Garcia GS, Sosa H, Searcy C, Vargas D, Janssen PL, Perez GM, Tschiffely AE, Janssen WG et al (2017) Lack of chronic neuroinflammation in the absence of focal hemorrhage in a rat model of low-energy blast-induced TBI. Acta Neuropathol Commun 5:80. https://doi. org/10.1186/s40478-017-0483-z

34. Gama Sosa MA, Gasperi RD, Rocher AB, Wang AC, Janssen WG, Flores $T$, Perez GM, Schmeidler J, Dickstein DL, Hof PR et al (2010) Age-related vascular pathology in transgenic mice expressing presenilin 1-associated familial Alzheimer's disease mutations. Am J Pathol 176:353-368. https://doi. org/10.2353/ajpath.2010.090482

35. Gandy S, Ikonomovic MD, Mitsis E, Elder G, Ahlers ST, Barth J, Stone JR, DeKosky ST (2014) Chronic traumatic encephalopathy: clinical-biomarker correlations and current concepts in pathogenesis. Mol Neurodegener 9:37. https://doi.org/10.1186/1750-1326-9-37

36. Garman RH, Jenkins LW, Switzer RC 3rd, Bauman RA, Tong LC, Swauger PV, Parks SA, Ritzel DV, Dixon CE, Clark RS et al (2011) Blast exposure in rats with body shielding is characterized primarily by diffuse axonal injury. J Neurotrauma 28:947-959

37. Goldstein LE, Fisher AM, Tagge CA, Zhang XL, Velisek L, Sullivan JA, Upreti C, Kracht JM, Ericsson M, Wojnarowicz MW et al (2012) Chronic traumatic encephalopathy in blast-exposed military veterans and a blast neurotrauma mouse model. Sci Transl Med 4:134ra160

38. Haghighi F, Ge Y, Chen S, Xin Y, Umali MU, De Gasperi R, Gama Sosa MA, Ahlers ST, Elder GA (2015) Neuronal DNA methylation profiling of blastrelated traumatic brain injury. J Neurotrauma 32:1200-1209. https://doi.org/ 10.1089/neu.2014.3640

39. Hald ES, Alford PW (2014) Smooth muscle phenotype switching in blast traumatic brain injury-induced cerebral vasospasm. Transl Stroke Res 5:385393. https://doi.org/10.1007/s12975-013-0300-3

40. Hamel E (2006) Perivascular nerves and the regulation of cerebrovascular tone. J Appl Physiol (1985) 100:1059-1064. https://doi.org/10.1152/ japplphysiol.00954.2005

41. Hjelle OP, Chaudhry FA, Ottersen OP (1994) Antisera to glutathione: characterization and immunocytochemical application to the rat cerebellum. Eur J Neurosci 6:793-804

42. Huber BR, Meabon JS, Hoffer ZS, Zhang J, Hoekstra JG, Pagulayan KF, McMillan PJ, Mayer CL, Banks WA, Kraemer BC et al (2016) Blast exposure causes dynamic microglial/macrophage responses and microdomains of brain microvessel dysfunction. Neuroscience 319:206-220. https://doi.org/10 1016/j.neuroscience.2016.01.022

43. ladecola C (2017) The neurovascular unit coming of age: a journey through neurovascular coupling in health and disease. Neuron 96:17-42. https://doi. org/10.1016/j.neuron.2017.07.030

44. Janssen WG, Vissavajjhala P, Andrews G, Moran T, Hof PR, Morrison JH (2005) Cellular and synaptic distribution of NR2A and NR2B in macaque monkey and rat hippocampus as visualized with subunit-specific monoclonal antibodies. Exp Neurol 191(Suppl 1):S28-S44. https://doi.org/10. 1016/j.expneurol.2004.08.020

45. Kabu S, Jaffer H, Petro M, Dudzinski D, Stewart D, Courtney A, Courtney M, Labhasetwar V (2015) Blast-associated shock waves result in increased brain vascular leakage and elevated ROS levels in a rat model of traumatic brain injury. PLoS One 10:e0127971. https://doi.org/10.1371/journal.pone.0127971

46. Kaur C, Singh J, Lim MK, Ng BL, Yap EP, Ling EA (1997) Ultrastructural changes of macroglial cells in the rat brain following an exposure to a nonpenetrative blast. Ann Acad Med Singap 26:27-29

47. Kondo A, Shahpasand K, Mannix R, Qiu J, Moncaster J, Chen CH, Yao Y, Lin YM, Driver JA, Sun Y et al (2015) Antibody against early driver of neurodegeneration cis P-tau blocks brain injury and tauopathy. Nature 523: 431-436. https://doi.org/10.1038/nature14658

48. Kuehn R, Simard PF, Driscoll I, Keledjian K, Ivanova S, Tosun C, Williams A, Bochicchio G, Gerzanich V, Simard JM (2011) Rodent model of direct cranial blast injury. J Neurotrauma 28:2155-2169

49. Wang JM, Chen J (2016) Damage of vascular endothelial barrier induced by explosive blast and its clinical significance. Chin J Traumatol 19: 125-128

50. Lange RT, Brickell TA, Ivins B, Vanderploeg RD, French LM (2013) Variable, not always persistent, postconcussion symptoms after mild TBI in U.S. military service members: a five-year cross-sectional outcome study. J Neurotrauma 30:958-969. https://doi.org/10.1089/neu.2012.2743

51. Li BC, Li Y, Xu C, Wang J, Chen Z, Li G, Zhang J, Hu S, Wang L, Feng H (2014) Blast-induced traumatic brain injury of goats in confined space. Neurol Res. https://doi.org/10.1179/1743132813Y.0000000314

52. Logsdon AF, Lucke-Wold BP, Turner RC, Li X, Adkins CE, Mohammad AS, Huber JD, Rosen CL, Lockman PR (2017) A mouse model of focal vascular injury induces astrocyte reactivity, tau oligomers, and aberrant behavior. Arch Neurosci 4(2): e44254

53. Logsdon AF, Meabon JS, Cline MM, Bullock KM, Raskind MA, Peskind ER, Banks WA, Cook DG (2018) Blast exposure elicits blood-brain barrier disruption and repair mediated by tight junction integrity and nitric oxide dependent processes. Sci Rep 8:11344. https://doi.org/10.1038/s41598-018-29341-6

54. Logsdon AF, Turner RC, Lucke-Wold BP, Robson MJ, Naser ZJ, Smith KE, Matsumoto RR, Huber JD, Rosen CL (2014) Altering endoplasmic reticulum stress in a model of blast-induced traumatic brain injury controls cellular fate and ameliorates neuropsychiatric symptoms. Front Cell Neurosci 8:421. https://doi.org/10.3389/fncel.2014.00421

55. Lu J, Ng KC, Ling G, Wu J, Poon DJ, Kan EM, Tan MH, Wu YJ, Li P, Moochhala $S$ et al (2012) Effect of blast exposure on the brain structure and cognition in Macaca fascicularis. J Neurotrauma 29:1434-1454. https://doi. org/10.1089/neu.2010.1591

56. Lucke-Wold BP, Logsdon AF, Smith KE, Turner RC, Alkon DL, Tan Z, Naser ZJ, Knotts CM, Huber JD, Rosen CL (2015) Bryostatin-1 restores blood brain 
barrier integrity following blast-induced traumatic brain injury. Mol Neurobiol 52:1119-1134. https://doi.org/10.1007/s12035-014-8902-7

57. Lucke-Wold BP, Naser ZJ, Logsdon AF, Turner RC, Smith KE, Robson MJ, Bailes JE, Lee JM, Rosen CL, Huber JD (2015) Amelioration of nicotinamide adenine dinucleotide phosphate-oxidase mediated stress reduces cell death after blast-induced traumatic brain injury. Transl Res 166:509-528 e501. https://doi.org/10.1016/j.trsl.2015.08.005

58. Ma Y, Xia X, Cheng JM, Kuang YQ, Yang T, Yang LB, Fan K, Gu JW (2014) Emodin inhibits inducible nitric oxide synthase in a rat model of craniocerebral explosive injury. Neurochem Res 39:1809-1816. https://doi, org/10.1007/s1 1064-014-1395-y

59. Mackie AR, Losordo DW (2011) CD34-positive stem cells: in the treatment of heart and vascular disease in human beings. Tex Heart Inst J 38:474-485

60. Meabon JS, Huber BR, Cross DJ, Richards TL, Minoshima S, Pagulayan KF, Li G, Meeker KD, Kraemer BC, Petrie EC et al (2016) Repetitive blast exposure in mice and combat veterans causes persistent cerebellar dysfunction. Sci Transl Med 8:321ra326. https://doi.org/10.1126/scitransImed.aaa9585

61. Mishra V, Skotak M, Schuetz H, Heller A, Haorah J, Chandra N (2016) Primary blast causes mild, moderate, severe and lethal TBI with increasing blast overpressures: experimental rat injury model. Sci Rep 6:26992. https://doi. org/10.1038/srep26992

62. Pasquinelli G, Tazzari PL, Vaselli C, Foroni L, Buzzi M, Storci G, Alviano F, Ricci F, Bonafe M, Orrico C et al (2007) Thoracic aortas from multiorgan donors are suitable for obtaining resident angiogenic mesenchymal stromal cells. Stem Cells 25:1627-1634. https://doi.org/10.1634/stemcells.2006-0731

63. Paulson OB, Strandgaard S, Edvinsson L (1990) Cerebral autoregulation. Cerebrovasc Brain Metab Rev 2:161-192

64. Perez-Garcia G, De Gasperi R, Gama Sosa MA, Perez GM, Otero-Pagan A, Tschiffely A, McCarron RM, Ahlers ST, Elder GA, Gandy S (2018) PTSD-related behavioral traits in a rat model of blast-induced $\mathrm{mTBl}$ are reversed by the mGluR2/3 receptor antagonist BCl-838. eNeuro 5. https://doi.org/10.1523/ ENEURO.0357-17.2018

65. Perez-Garcia G, Gama Sosa MA, De Gasperi R, Lashof-Sullivan M, MaudlinJeronimo E, Stone JR, Haghighi F, Ahlers ST, Elder GA (2016) Exposure to a predator scent induces chronic behavioral changes in rats previously exposed to low-level blast: implications for the relationship of blast-related TBI to PTSD. Front Neurol 7:176. https://doi.org/10.3389/fneur.2016.00176

66. Perez-Garcia G, Gama Sosa MA, De Gasperi R, Lashof-Sullivan M, MaudlinJeronimo E, Stone JR, Haghighi F, Ahlers ST, Elder GA (2018) Chronic posttraumatic stress disorder-related traits in a rat model of low-level blast exposure. Behav Brain Res 340:117-125. https:/doi.org/10.1016/j.bbr.2016.09.061

67. Perez-Garcia G, Gama Sosa MA, De Gasperi R, Tschiffely AE, McCarron RM, Hof PR, Gandy S, Ahlers ST, Elder GA (2018) Blast-induced "PTSD": evidence from an animal model. Neuropharmacology: Doi https://doi.org/10.1016/j. neuropharm.2018.09.023

68. Pun PB, Kan EM, Salim A, Li Z, Ng KC, Moochhala SM, Ling EA, Tan MH, Lu J (2011) Low level primary blast injury in rodent brain. Front Neurol 2:19. https://doi.org/10.3389/fneur.2011.00019

69. Rafaels KA, Bass CR, Panzer MB, Salzar RS, Woods WA, Feldman SH, Walilko T, Kent RW, Capehart BP, Foster JB et al (2012) Brain injury risk from primary blast. J Trauma Acute Care Surg 73:895-901. https://doi.org/10.1097/TA. 0b013e31825a760e

70. Rajkowska G, Hughes J, Stockmeier CA, Javier Miguel-Hidalgo J, Maciag D (2013) Coverage of blood vessels by astrocytic endfeet is reduced in major depressive disorder. Biol Psychiatry 73:613-621. https://doi.org/10.1016/j. biopsych.2012.09.024

71. Readnower RD, Chavko M, Adeeb S, Conroy MD, Pauly JR, McCarron RM, Sullivan PG (2010) Increase in blood-brain barrier permeability, oxidative stress, and activated microglia in a rat model of blast-induced traumatic brain injury. J Neurosci Res 88:3530-3539. https://doi.org/10.1002/jnr.22510

72. Reneer DV, Hisel RD, Hoffman JM, Kryscio RJ, Lusk BT, Geddes JW (2011) A multi-mode shock tube for investigation of blast-induced traumatic brain injury. J Neurotrauma 28:95-104

73. Rodriguez UA, Zeng Y, Deyo D, Parsley MA, Hawkins BE, Prough DS, DeWitt DS (2018) Effects of mild blast traumatic brain injury on cerebral vascular, histopathological, and Behavioral Outcomes in Rats. J Neurotrauma 35:375392. https://doi.org/10.1089/neu.2017.5256

74. Rubovitch $\mathrm{V}$, Ten-Bosch M, Zohar O, Harrison CR, Tempel-Brami C, Stein E, Hoffer BJ, Balaban CD, Schreiber S, Chiu WT et al (2011) A mouse model of blast-induced mild traumatic brain injury. Exp Neurol 232:280-289
75. Sajja VS, Tenn C, McLaws $L$, Vandevord PJ (2012) A temporal evaluation of cytokines in rats after blast exposure. Biomed Sci Instrum 48:374-379

76. Saljo A, Arrhen F, Bolouri H, Mayorga M, Hamberger A (2008) Neuropathology and pressure in the pig brain resulting from low-impulse noise exposure. J Neurotrauma 25:1397-1406

77. Searcy JL, Le Bihan T, Salvadores N, McCulloch J, Horsburgh K (2014) Impact of age on the cerebrovascular proteomes of wild-type and Tg-SwDI mice. PLoS One 9:e89970. https://doi.org/10.1371/journal.pone.0089970

78. Sharrock AE, Remick KN, Midwinter MJ, Rickard RF (2018) Combat vascular injury: influence of mechanism of injury on outcome. Injury. https://doi.org/ 10.1016/j.injury.2018.06.037

79. Shively SB, Horkayne-Szakaly I, Jones RV, Kelly JP, Armstrong RC, Perl DP (2016) Characterisation of interface astroglial scarring in the human brain after blast exposure: a post-mortem case series. Lancet Neurol 15:944-953. https://doi.org/10.1016/S1474-4422(16)30057-6

80. Simard JM, Pampori A, Keledjian K, Tosun C, Schwartzbauer G, Ivanova S, Gerzanich V (2014) Exposure of the thorax to a sublethal blast wave causes a hydrodynamic pulse that leads to perivenular inflammation in the brain. J Neurotrauma 31:1292-1304. https://doi.org/10.1089/neu.2013.3016

81. Skotak M, Wang F, Alai A, Holmberg A, Harris S, Switzer RC, Chandra N (2013) Rat injury model under controlled field-relevant primary blast conditions: acute response to a wide range of peak overpressures. Neurotrauma 30:1147-1160. https://doi.org/10.1089/neu.2012.2652

82. Stenmark KR, Yeager ME, El Kasmi KC, Nozik-Grayck E, Gerasimovskaya EV, Li M, Riddle SR, Frid MG (2013) The adventitia: essential regulator of vascular wall structure and function. Annu Rev Physiol 75:23-47. https://doi.org/10. 1146/annurev-physiol-030212-183802

83. Stokum JA, Keledjian K, Hayman E, Karimy JK, Pampori A, Imran Z, Woo SK, Gerzanich V, Simard JM (2017) Glibenclamide pretreatment protects against chronic memory dysfunction and glial activation in rat cranial blast traumatic brain injury. Behav Brain Res 333:43-53. https://doi.org/10.1016/j. bbr.2017.06.038

84. Svetlov SI, Prima V, Kirk DR, Gutierrez H, Curley KC, Hayes RL, Wang KK (2010) Morphologic and biochemical characterization of brain injury in a model of controlled blast overpressure exposure. J Trauma 69:795-804

85. Tchantchou F, Puche AA, Leiste U, Fourney W, Blanpied TA, Fiskum G (2018) Rat model of brain injury to occupants of vehicles targeted by land mines: mitigation by elastomeric frame designs. J Neurotrauma 35:1 192-1203. https://doi.org/10.1089/neu.2017.5401

86. Toklu HZ, Muller-Delp J, Yang Z, Oktay S, Sakarya Y, Strang K, Ghosh P, Delp MD, Scarpace PJ, Wang KK et al (2015) The functional and structural changes in the basilar artery due to overpressure blast injury. J Cereb Blood Flow Metab 35:1950-1956. https://doi.org/10.1038/jcbfm.2015.151

87. Turner RC, Naser ZJ, Logsdon AF, DiPasquale KH, Jackson GJ, Robson MJ, Gettens RT, Matsumoto RR, Huber JD, Rosen CL (2013) Modeling clinically relevant blast parameters based on scaling principles produces functional \& histological deficits in rats. Exp Neurol 248:520-529. https://doi.org/10.1016/ j.expneurol.2013.07.008

88. van Lookeren Campagne M, Oestreicher AB, van der Krift TP, Gispen WH, Verkleij AJ (1991) Freeze-substitution and Lowicryl HM20 embedding of fixed rat brain: suitability for immunogold ultrastructural localization of neural antigens. J Histochem Cytochem 39:1267-1279. https://doi.org/10. $1177 / 39.9 .1833448$

89. Wang Y, Wei Y, Oguntayo S, Wilder D, Tong L, Su Y, Gist I, Arun P, Long JB (2017) Cerebrospinal fluid chemokine (C-C motif) ligand 2 is an earlyresponse biomarker for blast-overpressure-wave-induced Neurotrauma in rats. J Neurotrauma 34:952-962. https://doi.org/10.1089/neu.2016.4465

90. Wang Y, Wei Y, Oguntayo S, Wilkins W, Arun P, Valiyaveettil M, Song J, Long JB, Nambiar MP (2011) Tightly coupled repetitive blast-induced traumatic brain injury: development and characterization in mice. J Neurotrauma 28 2171-2183

91. Wolf SJ, Bebarta VS, Bonnett CJ, Pons PT, Cantrill SV (2009) Blast injuries. Lancet 374:405-415

92. Yeoh S, Bell ED, Monson KL (2013) Distribution of blood-brain barrier disruption in primary blast injury. Ann Biomed Eng 41:2206-2214. https:// doi.org/10.1007/s10439-013-0805-7

93. Zhang Y, Yang Y, Tang H, Sun W, Xiong X, Smerin D, Liu J (2014) Hyperbaric oxygen therapy ameliorates local brain metabolism, brain edema and inflammatory response in a blast-induced traumatic brain injury model in rabbits. Neurochem Res 39:950-960. https://doi.org/10.1007/s1 1064-014-1292-4 